\title{
Progress in Borneol Intervention for Ischemic Stroke: A Systematic Review
}

Yong Li, Mihong Ren, Jiajun Wang, Rong Ma, Hai Chen, Qian Xie, Hongyan Li, Jinxiu Li and Jian Wang *

College of Pharmacy, Chengdu University of Traditional Chinese Medicine, Chengdu, China

Background: Borneol is a terpene and bicyclic organic compound that can be extracted from plants or chemically synthesized. As an important component of proprietary Chinese medicine for the treatment of stroke, its neuroprotective effects have been confirmed in many experiments. Unfortunately, there is no systematic review of these studies. This study aimed to systematically examine the neuroprotective effects of borneol in the cascade reaction of experimental ischemic stroke at different periods.

Methods: Articles on animal experiments and cell-based research on the actions of borneol against ischemic stroke in the past $20^{\circ}$ years were collected from Google Scholar, Web of Science, PubMed, ScienceDirect, China National Knowledge Infrastructure (CNKI), and other biomedical databases. Meta-analysis was performed on key indicators in vivo
OPEN ACCESS

Edited by:

Jiahong Lu,

University of Macau,

China

Reviewed by:

He-Hui Xie,

Shanghai Jiao Tong University, China

Ziying Wang,

Jinan University, China

*Correspondence: Jian Wang jianwang08@163.com

Specialty section: This article was submitted to

Ethnopharmacology,

a section of the journal

Received: 15 September 2020 Accepted: 14 April 2021

Published: 04 May 2021

Citation:

Li Y, Ren M, Wang J, Ma R, Chen H, Progress in Borneol Intervention for Ischemic Stroke: A Systematic Review.

Front. Pharmacol. 12:606682. doi: 10.3389/fphar.2021.606682
Frontiers in Pharmacology Xie Q, Li H, Li J and Wang J (2021) experiments. After sorting the articles, we focused on the neuroprotective effects and mechanism of action of borneol at different stages of cerebral ischemia.

Results: Borneol is effective in the prevention and treatment of nerve injury in ischemic stroke. Its mechanisms of action include improvement of cerebral blood flow, inhibition of neuronal excitotoxicity, blocking of $\mathrm{Ca}^{2+}$ overload, and resistance to reactive oxygen species injury in the acute ischemic stage. In the subacute ischemic stage, borneol may antagonize blood-brain barrier injury, intervene in inflammatory reactions, and prevent neuron excessive death. In the late stage, borneol promotes neurogenesis and angiogenesis in the treatment of ischemic stroke.

Conclusion: Borneol prevents neuronal injury after cerebral ischemia via multiple action mechanisms, and it can mobilize endogenous nutritional factors to hasten repair and regeneration of brain tissue. Because the neuroprotective effects of borneol are mediated by various therapeutic factors, deficiency caused by a single-target drug is avoided. Besides, borneol promotes other drugs to pass through the blood-brain barrier to exert synergistic therapeutic effects.

Keywords: L-borneol, D-borneol, DL-borneol, ischemic stroke, cascade reaction, neuroprotection

\section{INTRODUCTION}

Borneol is a bicyclic terpenoid with strong fat solubility (Lv et al., 2012). The 2020 edition of Chinese Pharmacopoeia contains three commercial borneol products: D-borneol (Chinese name "Tianranbingpian"), L-borneol (Chinese name "Aipian"), and Synthetic borneol (Chinese name "Bingpian"). D-borneol are extracted from fresh branches and leaves of Cinnamomum camphora (L.) 


\section{Summary of Borneol against Ischemic Stroke}

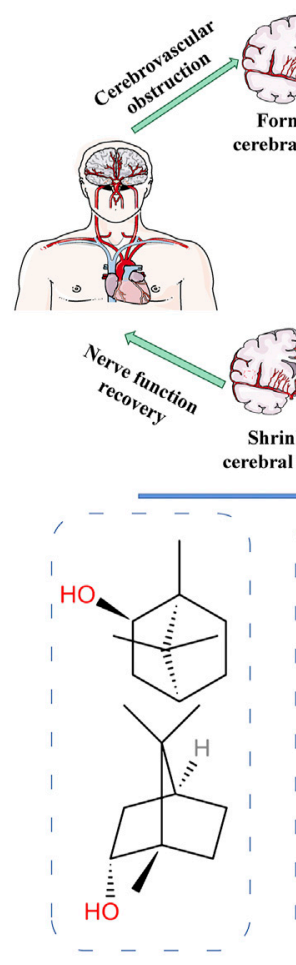

A L-borneol

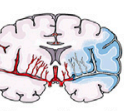

Formation of Formation of
cerebral infarction Q
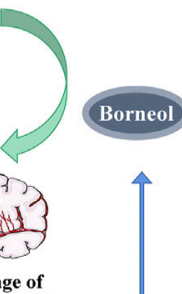
Borneol

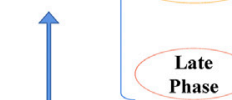

Improving cerebral blood flow

Inhibition of neuronal excitotoxicity

Suppression of $\mathrm{Ca}^{2+}$ overload

> Protection against ROS damage

> Regulation of the expression of NOS

$>$ Suppress acute hyperthermia

$>$ Protect the blood-brain barrier

$>$ Attenuate inflammatory response

$>$ Hinder nerve cell death

Promote neurogenesis.

Stimulate angiogenesis

FIGURE 1 | Mechanism of borneol against cerebral ischemia injury and chemical structure of three borneols. (A) Levorotatory borneol (endo-(1 S)-1,7,7-trimethylbicyclo [2.2.1] heptan-2-ol, (-)-borneol), which is extracted from fresh leaves of Blumea balsamifera (L.) DC. (B) Dextrorotatory borneol (endo-(1R)-1,7,7-trimethylbicyclo [2.2.1] heptan-2-ol, (+)-borneol), which is extracted from fresh branches and leaves of Cinnamomum camphora (L.) Presl. (C) Synthetic borneol (DL-borneol) is an optically inactive $( \pm)$ borneol that is mainly a mixture of $( \pm)$ borneol and is obtained via the chemical transformation of camphor and turpentine oil.
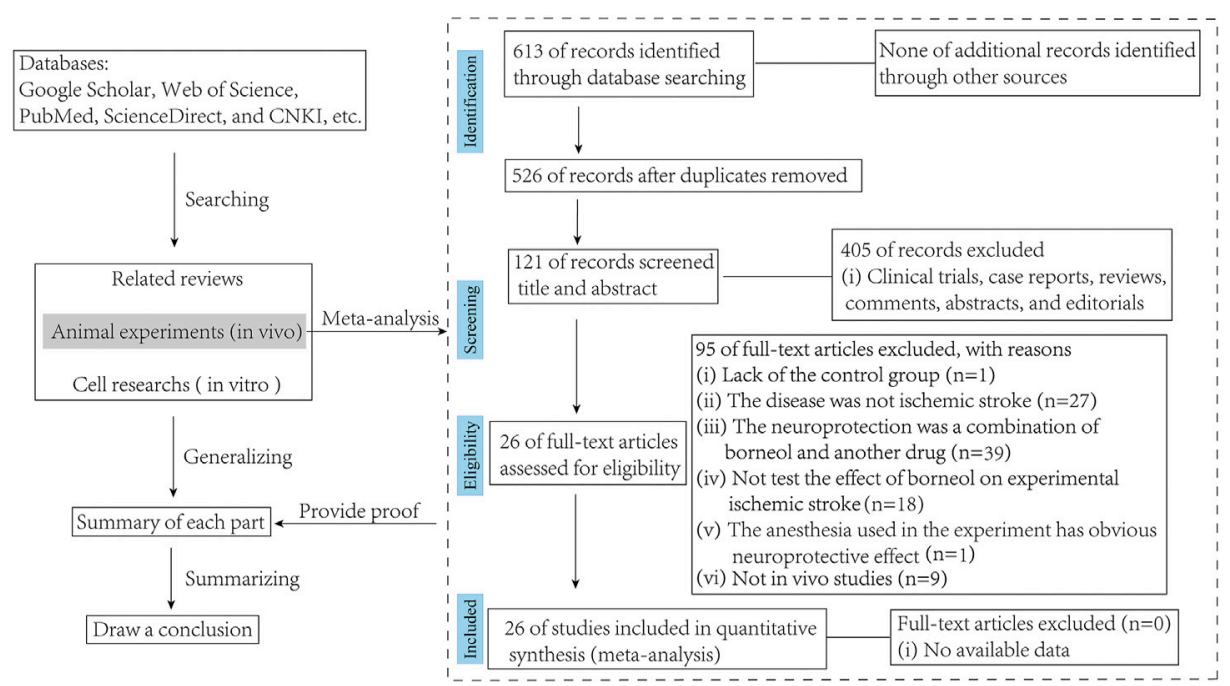

FIGURE 2 | Flow diagram of the search process. 
Presl.or Dryobalanops aromatica Gaertn.f (Yang et al., 2018; Wang and Wang, 2018). L-borneol is processed from the sublimation of Blumea balsamifera (L.) DC (Islam et al., 2010). Synthetic borneol is a racemic borneol (A mixture with D-borneol and L-borneol as the main components) prepared by chemical synthesis of turpentine and camphor (Chinese Pharmacopoeia Committee, 2020) (Figure 1). Borneol has been used in China for more than 1600 years (Li et al., 2013). In Traditional Chinese Medicine (TCM) theory, borneol is a upper ushering drug that guides herbs to their target organs, especially in the upper part of the body, including the brain (Chen et al., 2019). In addition, borneol is suitable for the treatment of mental diseases accompanied by signs of heat syndrome because the herbal medicine has the property of being "cold" (Zou et al., 2017; Wang and Wang, 2018). Several proprietary Chinese medicine preparations such as Angong Niuhuang pills and Xingnaojing injection contain borneol and are widely used in the clinical treatment of stroke (Guo et al., 2014; Zhang et al., 2019).

Cerebral stroke is a disorder of cerebral blood circulation of the central nervous system and may either be ischemic or hemorrhagic. Ischemic stroke accounts for approximately $80 \%$ of stroke cases (Fan et al., 2017). Ischemic stroke is accompanied by extremely complex physiological and pathological processes. The acute stage of ischemic stroke is characterized by glutamate excitotoxicity, intracellular $\mathrm{Ca}^{2+}$ overload, oxidative stress, and production of free radicals. The subacute stage is characterized by apoptosis and necrosis, blood-brain barrier damage, brain edema, and an inflammatory response. The late stage of ischemic stroke is characterized by reactive astrocyte proliferation, glial scar formation, angiogenesis, and neurogenesis (Steliga et al., 2020). These processes occur at different time points, overlap with each other, and eventually lead to brain injury and repair after ischemia.

In the study of brain diseases, the regulatory effect of borneol on blood-brain barrier has always been the focus of relevant practitioners. In fact, there are also abundant studies have shown that borneol has a neuroprotective effect on cerebral ischemic. Unfortunately, the neuroprotective mechanism of borneol is complex and involves many aspects, and only few reviews have focused on the protective mechanism of borneol at different periods of cerebral ischemia. Therefore, we take the review of borneol intervention in cerebral ischemia as the main content of the article, and supplement the meta-analysis of key indicators in vivo studies, hoping to provide some valuable references for borneol's experimental research and clinical application.

\section{METHODS}

\section{Search Strategy}

A comprehensive search strategy was conducted in several databases, including Google Scholar, Web of Science, ScienceDirect, PubMed, and CNKI from their inceptions to March 2021. For data mining, the following words were used in the databases mentioned above: "borneol" or "D-borneol" or
"L-borneol" or "synthetic borneol" and "cerebral ischemic" or "ischemic stroke" or "neuroprotection." In almost all cases, the original articles or abstracts were obtained and the relevant data was extracted.

\section{Eligibility and Exclusion Criteria}

Both animal experiments (in vivo) and cell studies (in vitro) on borneol intervention in ischemic stroke have been included in the review. But all studies accepted for meta-analysis should be met the following eligibility criteria: 1) The drugs used must be borneol, whether D-borneol, L-borneol, or synthetic borneol, it should be noted that the literature on combination of drugs, which sets borneol alone as a group to compare with the model group, can also be included in this study; 2) The animal model used must be a cerebral ischemia model, whether permanent or cerebral ischemia-reperfusion; and 3) the control group receiving vehicle or no adjunct intervention was included in the studies. Exclusion criteria were as follows: 1) the study was a case report, clinical trial, review, or in vitro study; 2) lack of the control group; 3) the targeting disease was not ischemic stroke; 4) the intervention was a combination of borneol and another agent with potential effect on ischemic stroke; 5) the anesthesia used in the experiment has obvious neuroprotective effect (Figure 2).

\section{Statistical Analysis}

To evaluate the effect of borneol on cerebral ischemia in animal experiments, Revman version 5.3 was performed for statistical analysis. If statistical heterogeneity was found $\left(p<0.1, I^{2}>50 \%\right)$, a model of random effect (RE) was applied to evaluate pooled effect with $95 \% \mathrm{CI}$; and if no statistical evidence of heterogeneity existed ( $p \geq 0.1, I^{2} \leq 50 \%$ ), a model of fixed effect (FE) was set with $95 \%$ CI. If the outcomes were applied at the same scale, the weighted mean difference (WMD) was calculated as a summary statistic; and if the same results were measured in different ways, the standardized mean differences (SMD) were used. Heterogeneity was assessed by standard chi-square test and $I^{2}$ statistics. A probability value less than 0.05 was considered statistically significant. To minimize bias and human error, the meta-analysis was performed by 2 independent reviewers and disagreements reconciled by a third independent reviewer.

\section{RESULTS}

\section{Mechanism of Borneol Intervention in the Acute Stage of Ischemic Stroke}

Decreased cerebral blood flow in the acute phase of ischemic stroke (within $24 \mathrm{~h}$ of human cerebral ischemia) causes a decrease in oxygen and metabolic substrates to neurons. Sbusequently, The lack of oxygen interrupts oxidative phosphorylation by the mitochondria and drastically reduces cellular ATP production. Inhibition of the $\mathrm{Na}^{+} / \mathrm{K}^{+}$-ATPase function causes a profound loss of ionic gradients and depolarization of regulated neurons, which leads to excessive release of excitatory amino acids-particularly glutamate-to the extracellular compartment. The presence of excessive amounts of free glutamate into the synapses and extrasynaptic sites can lead eventually to neuronal death. 


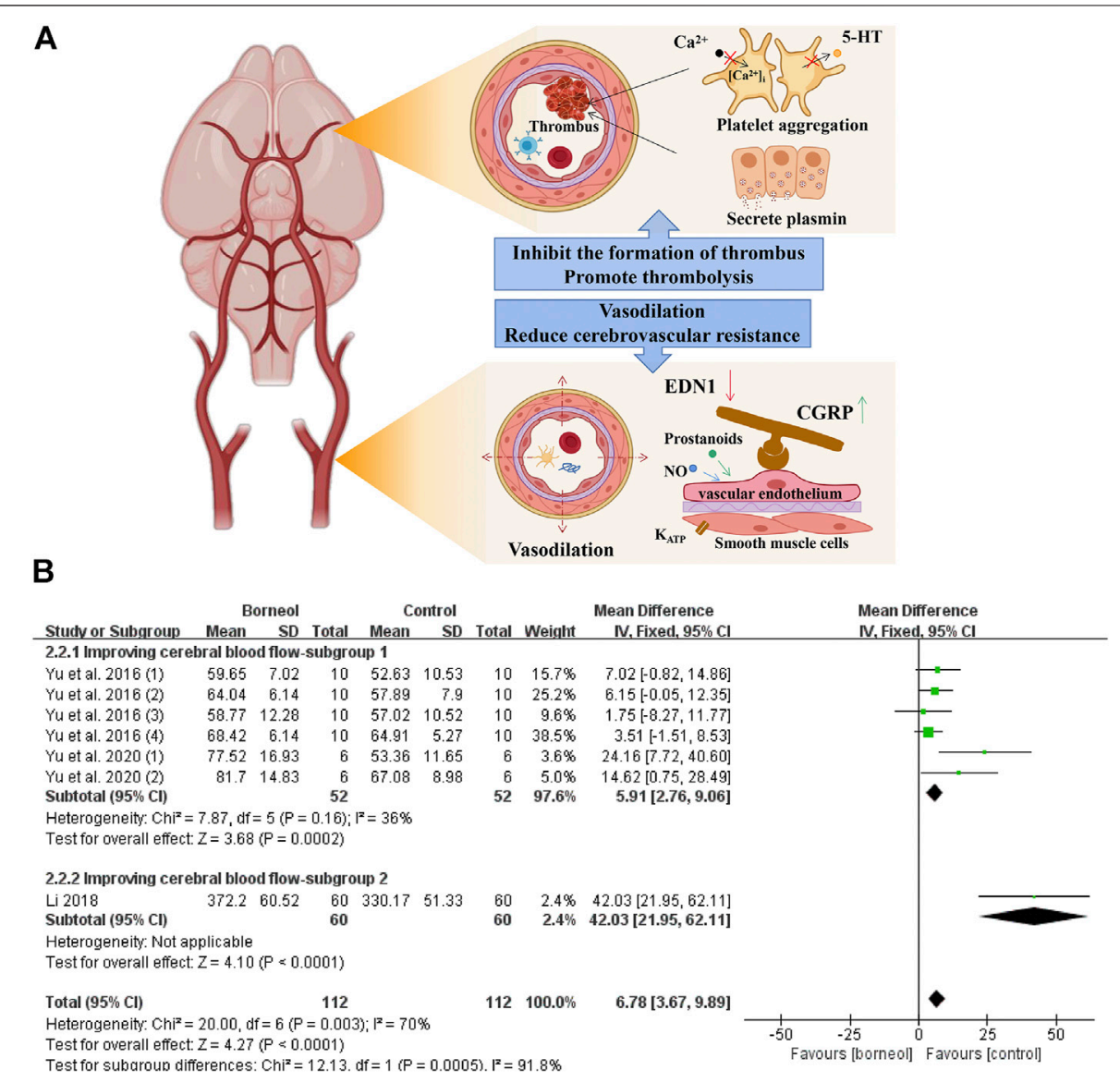

FIGURE 3 | (A) Borneol reduces platelet aggregation by blocking the release of $5-\mathrm{HT}$ and the increasing of intracellular $\mathrm{Ca}^{2+}$ levels, and interferes with thrombus formation by inhibiting thrombin activity. In addition, borneol activates the fibrinolytic system to promote the dissolution of plasma euglobulin. Borneol also improve cerebral blood flow under ischemia by regulating vascular neuropeptides. (B) The forest plots: the borneol group vs. the control group on cerebral blood flow. Subgroup 1 measures the cerebral blood flow in a certain brain area such as the hypothalamus or hippocampus in a tGCIR rodent model, showing that borneol significant increase of cerebral blood flow in the treatment of cerebral ischemic injury $\left(n_{T} / n_{C}=52 / 52, M D 5.91,95 \%\right.$ Cl: 2.76 9.06, $p=0.0002$; heterogeneity $\chi^{2}=7.87$, df $=5$, $P^{2}=36 \%$ ). In subgroup 2, the cerebral blood flow of bilateral parietal cortex was monitored, and the model was induced by photochemical method, which also showed that borneol could improve the cerebral blood flow.

Excitotoxicity leads to a number of deleterious consequences, including impairment of cellular calcium homeostasis, generation of free radicals and oxidative stress, mitochondrial damage, and activation of transcription factors. All these mechanisms' acting synergy can cause acute neuron death by apoptosis (Rama and Garcia, 2016). Borneol participates in multiple physiological and pathological processes in the acute phase of ischemic stroke and impedes disease progression by antagonizing the damage in the initial stage of ischemic stroke.

\section{Improving Cerebral Blood Flow}

Disordered energy metabolism is required in the development of cerebral ischemia. Stenosis and occlusion of blood vessels lead to the interruption of local blood flow and alters blood circulation. Cerebral infarction occurs when the supply of nutrients provided by local blood circulation does not meet the energy metabolism needs of brain cells beyond a certain time limit. Therefore, timely improvement of the blood supply to the brain is the primary goal of the clinical treatment of ischemic stroke. Borneol has the pharmacological effects of relaxing blood vessels, reducing blood pressure and cerebral vascular resistance, inhibiting thrombosis, and promoting thrombolysis, thereby improving cerebral blood flow after cerebral ischemia.

\section{Regulation of Vascular Neuropeptide}

In an in vivo canine study, DL-borneol reduced abdominal aortic blood pressure, increased common carotid blood flow, and reduced cerebrovascular resistance (Shang et al., 2015). L-borneol has a vasorelaxant effect that depends on the presence of vascular endothelium and the participation of nitric oxide (NO) and prostanoids. In addition, L-borneol directly relaxed vascular smooth muscle, which is dependent on $\mathrm{K}_{\mathrm{ATP}}$ channels (Santos et al., 2018). Under pathological conditions, D-borneol increased blood flow in the cortex in photochemical-mediated cerebral ischemia model rats and DLborneol increased blood flow in the cortex and striatum in transient global cerebral ischemia reperfusion (tGCIR) animal models (Yu et al., 2016; Li, 2018; Wang et al., 2018; Yu et al., 
2020). The meta-analysis results of three studies jointly suggested that borneol could improve the blood microcirculation in different brain regions (Figure 3).

Endothelin 1 (EDN1/ET) and calcitonin gene-related peptide (CGRP) are neuropeptides that contract and dilate blood vessels, respectively. Cerebral ischemia/reperfusion injury induces increased EDN1 secretion, promotes vascular smooth muscle contraction, and aggravates low perfusion. Conversely, CGRP is a strong neuropeptide vasodilator that serves as an endogenous endothelin antagonist to reverse vasospasms and improve blood circulation. Stroke leads to increased EDN1 and decreased CGRP levels (Yuan et al., 2006; Giannopoulos et al., 2008). Borneol can effectively reduce EDN1 levels and tends to increase CGRP levels (Huang, 2018), suggesting that it can dilate blood vessels and improve cerebral blood flow during cerebral ischemia.

Regulation of the Fibrinolytic System and Anti-Thrombosis The thrombus causing cerebral ischemia is mainly composed of platelets, leukocytes, erythrocytes, and fibrin (Michael et al., 2016). The dissolution time of plasma euglobulin reflects the activity of fibrinolytic enzymes. Borneol significantly shortens the euglobulin lysis time in rats, reduces the weight of the whole blood clot in mice, and inhibits ADP-induced platelet aggregation in rabbits (He, 2005). Furthermore, borneol inhibits venous thrombosis and arteriovenous shunt in a concentrationdependent manner and exerts anticoagulant activity by prolonging prothrombin time and thrombin time ( $\mathrm{Li}$ et al., 2008). 5-hydroxytryptamine (5-HT), secreted and released by platelets, plays an important role in platelet aggregation and thrombosis. $\mathrm{Ca}^{2+}$, as the second messenger, also plays a key role in the process of platelet activation. The deformation, aggregation and release of platelets are accompanied by the increase in the level of intracellular $\mathrm{Ca}^{2+}$ (Wöckel et al., 2008). Borneol inhibits $\mathrm{FeCl}_{3}$-induced arterial thrombosis in rats, which involves inhibition of platelet $5-\mathrm{HT}$ release and platelet aggregation, as well as the decrease of cytoplasmic $\mathrm{Ca}^{2+}$ level in platelet (Yang et al., 2010). The mechanisms of borneol improves cerebral blood flow and the results of meta-analysis of cerebral blood flow are shown in Figure 3.

\section{Inhibition of Neuronal Excitotoxicity}

The excessive release of glutamate and the drastic disruption of glutamate transporters, that occurs early after the cerebral ischemic is toxic to neurons, mainly through the activation of ionotropic receptors and intracellular calcium overload that trigger detrimental cascades causing excitotoxic neuronal death (Lai et al., 2014). Conversely, Some in vitro studies showed that activation of $\mathrm{GABA}_{\mathrm{A}}$ and $\mathrm{GABA}_{\mathrm{B}}$ receptors played a neuroprotective role (Costa et al., 2004; Xu et al., 2008). The expression of $\mathrm{GABA}_{\mathrm{A}}$ receptors after ischemia is decreased in distinct brain regions of rodents subjected to transient MCAO (Huang and Zhao, 2017). And the disruption of GABA-mediated neurotransmission early during reperfusion may also contribute to ongoing neuronal excitability (Mele et al., 2014). Neuroprotection is achieved in the preclinical setting by GABAergic drugs acting through multiple mechanisms, such as GABA receptors agonists or positive modulators. Borneol plays a neuroprotective role by reducing glutamate (Glu) levels, activating $\mathrm{GABA}_{\mathrm{A}}$ receptor, and then blocking the necrosis and apoptosis of neurons.

In one study, D-borneol and L-borneol enhanced the actions of $\gamma$-aminobutyric acid (GABA) at recombinant $\mathrm{GABA}_{\mathrm{A}}$ receptor and had moderate direct action on these receptors (Granger et al., 2005). D-borneol has neuroprotective effects after primary neuronal injury induced by glutamate at low concentrations, and this effect is consistent with the activation of $\mathrm{GABA}_{\mathrm{A}}$ receptor (Cheng et al., 2006; Chen et al., 2013). In addition, borneol reduces Glu levels in the hippocampus and hypothalamus in a tGCIR rat model (Yu et al., 2019a). The inhibitory effects of borneol on Glu secretion were also confirmed in a transient bilateral carotid occlusion (tBCO) rodent model (Huang, 2018). The meta-analysis results of the above two studies further suggested that borneol could reduce glutamate levels with small heterogeneity (Figure 4). Borneol promoted glutamate clearance in hypoxia/reoxygenation astrocytes and improved astrocyte viability during hypoxia (Chai et al., 2019). Borneol, combined with echinacoside, artificial moschus, Ligusticum chuanxiong, and other drugs, can also improve the content of Glu after cerebral ischemia (Liu et al., 2002a; Zhong et al., 2012; Yu et al., 2019b). The mechanisms by which borneol attenuates the toxicity of excitatory amino acid and the meta-analysis results of glutamate level are shown in Figure 4.

\section{Protection Against Damage Caused by Reactive Oxygen Species}

The reactive oxygen species (ROS) causing cerebral ischemic injury include superoxide anion $\left(\mathrm{O}^{2-}\right)$, hydroxyl radical $\left(\mathrm{OH}^{-}\right)$, and hydrogen peroxide $\left(\mathrm{H}_{2} \mathrm{O}_{2}\right)$. Brain tissue is rich in iron ions that catalyze the formation of free radicals, which makes the brain more vulnerable to ROS (González-Ibarra et al., 2011). When thrombolysis exceeds the therapeutic time window, a large amount of ROS is produced by dysfunctional mitochondria, the neutrophil respiratory burst, increased xanthine oxidase formation in capillary endothelial cells, and catecholamine self-oxidation after cerebral ischemia/reperfusion (Huang and Zhao, 2017). Furthermore, cerebral ischemia decreases the ability of the antioxidant system to scavenge ROS. The antioxidant system is composed of glutathione peroxidase (GSH-Px), superoxide dismutase (SOD), catalase (CAT), and other antioxidant enzymes. Improving antioxidant enzymes activity or activating related pathways is an important way to combat ischemic brain injury. Borneol improves the body's ability to resist ROS by increasing the activity of antioxidant enzymes and activating the nuclear factor erythroid 2-related factor 2 (Nrf2)antioxidant response element (ARE) signaling pathway.

Several studies have shown that borneol injection reduces malondialdehyde (MDA) levels and increases SOD and GSH-Px activity to accelerate the scavenging of superoxide anion free radicals after pMCAO in a rodent model (He et al., 2006; He et al., 2007). Borneol also increases SOD and GSH-Px activity and decreases MDA levels in the cerebral cortex, hippocampus, hypothalamus, and striatum of rats subjected to tGCIR (Yu et al., 2016). L-borneol and DL-borneol increase SOD and decrease MDA in ischemic brain tissue and the serum in a 


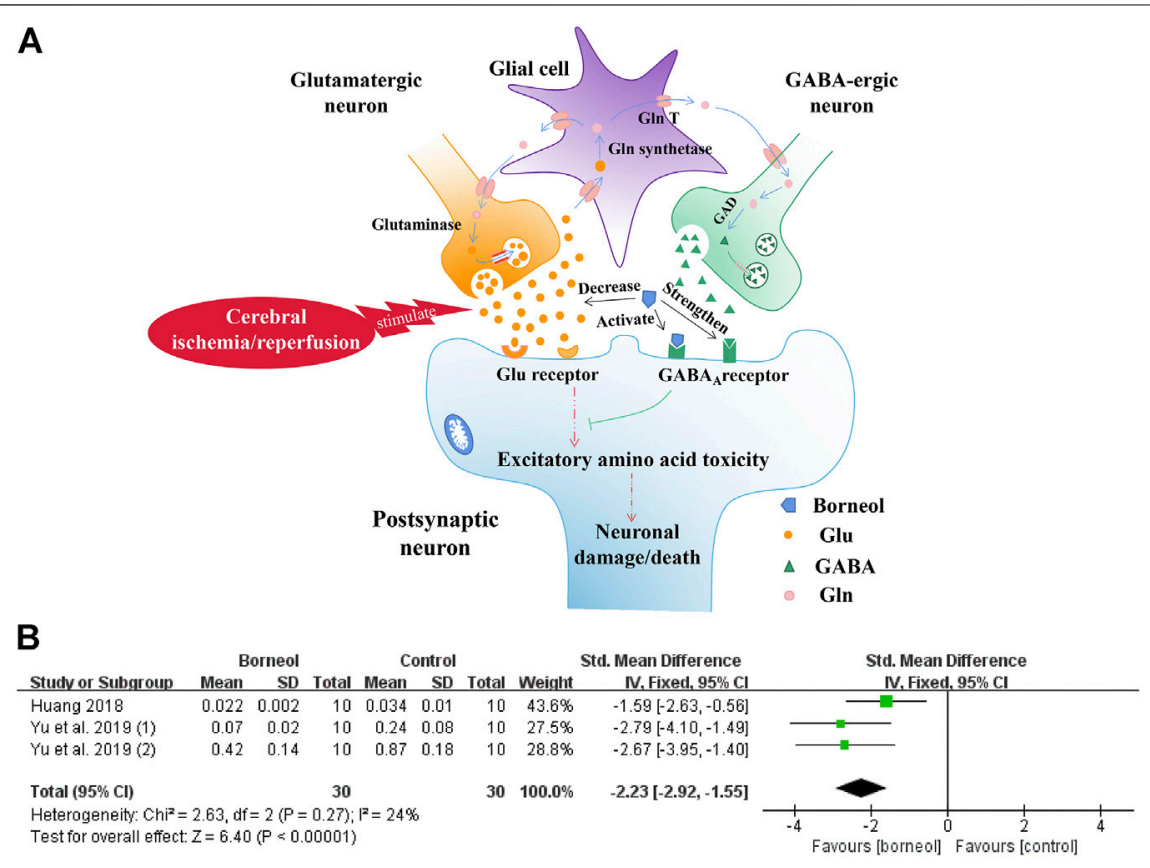

FIGURE 4 | (A) Borneol inhibits the increase of extracellular glutamate levels in the ischemic state and strengthens the binding of GABA to GABA receptors. The direct activation of the $\mathrm{GABA}_{\mathrm{A}}$ receptor by borneol could also inhibit glutamate-mediated excitatory amino acid toxicity. (B) The forest plots: the borneol group vs. the control group on glutamate levels. Meta-analysis of two studies with three comparisons showed that animals in the borneol group had statistically significant lower glutamate levels than the control group $\left(\mathrm{n}_{\mathrm{T}} / \mathrm{n}_{\mathrm{C}}=30 / 30, \mathrm{SMD}-2.23,95 \% \mathrm{Cl}:-2.92 \sim-1.55, p<0.00001\right.$, heterogeneity $\chi^{2}=2.63$, df $\left.=2, l^{2}=24 \%\right)$.

rodent model of tMCAO (Tian, 2013). The meta-analysis results of related studies further suggested that borneol could resist free radical damage by reducing MDA levels and increasing the activities of GSH-PX and SOD (Figure 5). Borneol, combined with musk, Ligusticum chuanxiong, and other drugs, can also attenuate ROS injury after cerebral ischemia (Liu et al., 2002b; Wang, 2011; Yu et al., 2017a). Besides, DL-borneol increases SOD and CAT activity, and D-Borneol increases SOD and GSH-Px activity in OGD/R-treated PC12 cells (Huang et al., 2020).

Borneol may protect against free radical damage by activating the Nrf2-ARE signaling pathway. Nrf2, an anti-oxidative stress nuclear transcription factor, translocates from the cytoplasm to the nucleus and binds to the ARE receptor to activate the Nrf2ARE signaling pathway when cells are exposed to ROS. The activation of this pathway induces the expression of downstream antioxidant enzymes to reduce cell damage caused by ROS and maintain the dynamic cellular redox balance (Wang et al., 2015). Circulating macrophages will enter the core area of cerebral ischemia through the blood-brain barrier to remove damaged cells after organic injury occurs in the brain. An in vitro cell experiment demonstrated that D-borneol promotes the expression of Nrf2 in RAW 264.7 macrophages stimulated with lipopolysaccharide (LPS) (Sun et al., 2019). It is also worth mentioning that L-borneol and D-borneol protected human neuroblastoma cells (SH-SY5Y) against $\beta$-amyloid induced toxicity, exerted an antioxidative effect by increaseing the expression and nuclear translocation of Nrf2 (Hur et al., 2013). Borneol, combined with Salvia miltiorrhiza, not only increased SOD levels and decreased MDA levels but also upregulated the expression of Nrf2 and inhibited the oxidative stress. The combination of these two drugs is more effective than Salvia miltiorrhiza alone, suggesting that borneol plays a synergistic role by activating the Nrf2-ARE signaling pathway in vivo (Liang et al., 2016). The actions of borneol against ROS damage and the meta-analysis results of related indicators are detailed in Figure 5.

\section{Regulation of the Expression of NOS to Protect Against NO Damage}

NO plays a dual role in neuroprotection and neurotoxicity. Nitric oxide synthase (NOS) isoforms are crucial in determining the role of NO in cerebral ischemia. There are three isoforms of NOS: neuronal NOS (nNOS/NOS1), inducible NOS (iNOS/NOS2), and endothelial NOS (eNOS/NOS3) (Chen et al., 2017). The transient increase of $\mathrm{NO}$ after cerebral ischemia is mainly mediated by eNOS and nNOS. NO synthesized by eNOS can dilate blood vessels, inhibit platelet aggregation, reduce leukocyte adhesion, and enhance collateral circulation to play a short-term neuroprotective effect (Ito et al., 2010). NO synthesized by nNOS participates in glutamate-induced neuronal $\mathrm{Ca}^{2+}$ overload, mediates early glutamate excitotoxicity (Zhou and Zhu, 2009). Also, nNOS activation contributes to microvascular damage and decreased cerebral perfusion early after reoxygenation and worsens brain damage (Hsu et al., 2014). The slow upregulation of iNOS in the late stage of cerebral ischemia leads to delayed neuronal injury by producing excessive NO, increasing microvascular permeability, and inducing brain edema (Liu and Mu, 2014). Therefore, 


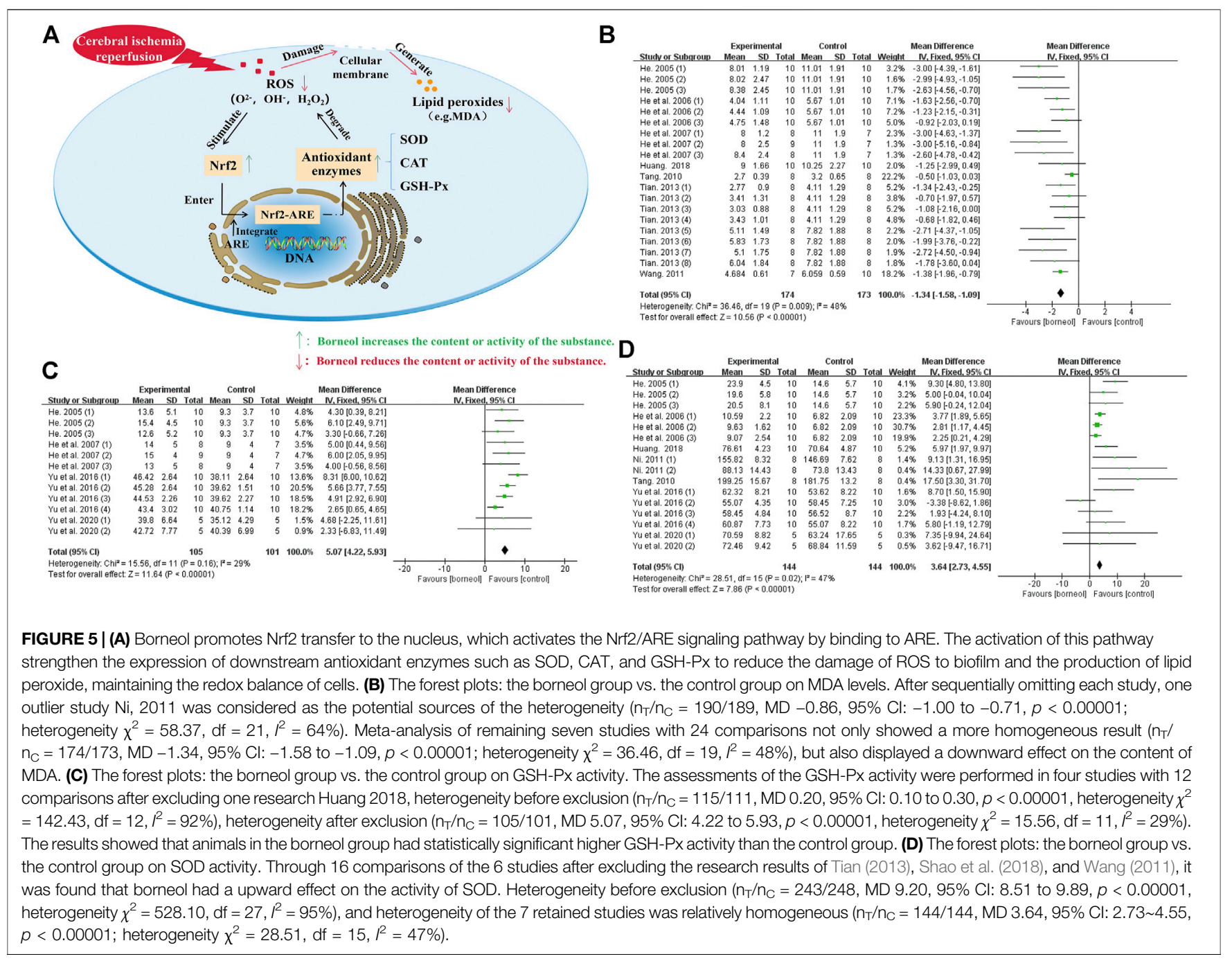

promoting the expression of eNOS in the early stage or inhibiting the expression of iNOS in the latter stage is a good therapeutic strategy for treating cerebral ischemia. Studies have found that borneol plays a neuroprotective effect by increasing eNOS expression and reducing iNOS expression, but the effect of borneol on nNOS is not clear.

Intravenous administration of D-borneol inhibits iNOS expression in rodent brain tissue and, consequently, reduces peroxynitrite $\left(\mathrm{ONOO}^{-}\right)$levels after tMCAO and pMCAO (Wu et al., 2014b; Chang et al., 2017). L-borneol and DL-borneol also reduce the expression level of iNOS in a pMCAO rodent model (Tian, 2013). In an in vitro oxygen-glucose deprivation-reperfusion (OGD/R) ischemia model, borneol inhibited the activity and expression of iNOS in primary cortical neurons and, therefore, reduced the production of iNOS-derived NO (Liu et al., 2011). In addition, borneol promotes the synthesis of eNOS-derived NO under both physiological and brain contusion conditions and has a stronger effect on the synthesis of NO by endothelial cells in the pathological state (Zhao et al., 2001). Borneol, combined with catalpol and puerarin, increases the expression of eNOS in rats with MCAO (Wu et al., 2016). The combination of borneol and musk promotes the synthesis of NO in vascular endothelial cells and inhibits the expression of iNOS in a global cerebral ischemia/ reperfusion rat model (Zhang et al., 2002; Liu et al., 2011).

\section{Inhibition of Intracellular $\mathrm{Ca}^{2+}$ Overload}

Calcium overload is considered being the last common pathway of neuronal injury during cerebral ischemia/reperfusion (Vannucci et al., 2001; Shi, 2014). The ways of calcium entering neurons following cerebral ischemia include glutamate receptors; voltage-dependent calcium channel; transient receptor potential channels; acid-sensing ion channels; sodium-calcium exchanger operating in entry mode; inward excitotoxic injury current calcium permeable channels; mitochondria and endoplasmic reticulum calcium release, etc. And the pathways of calcium exit into neurons include $\mathrm{Ca}^{2+}$ ATPase pump; $\mathrm{Na}^{+}-\mathrm{Ca}^{2+}$ exchanger operating in exit mode, etc (Singh et al., 2019).

It was found that both L-borneol and DL-borneol significantly reduced $\mathrm{Ca}^{2+}$ concentration in ischemic ipsilateral brain tissue of rats subjected to tMCAO (Ni, 2011; Tian, 2013). DL-borneol 


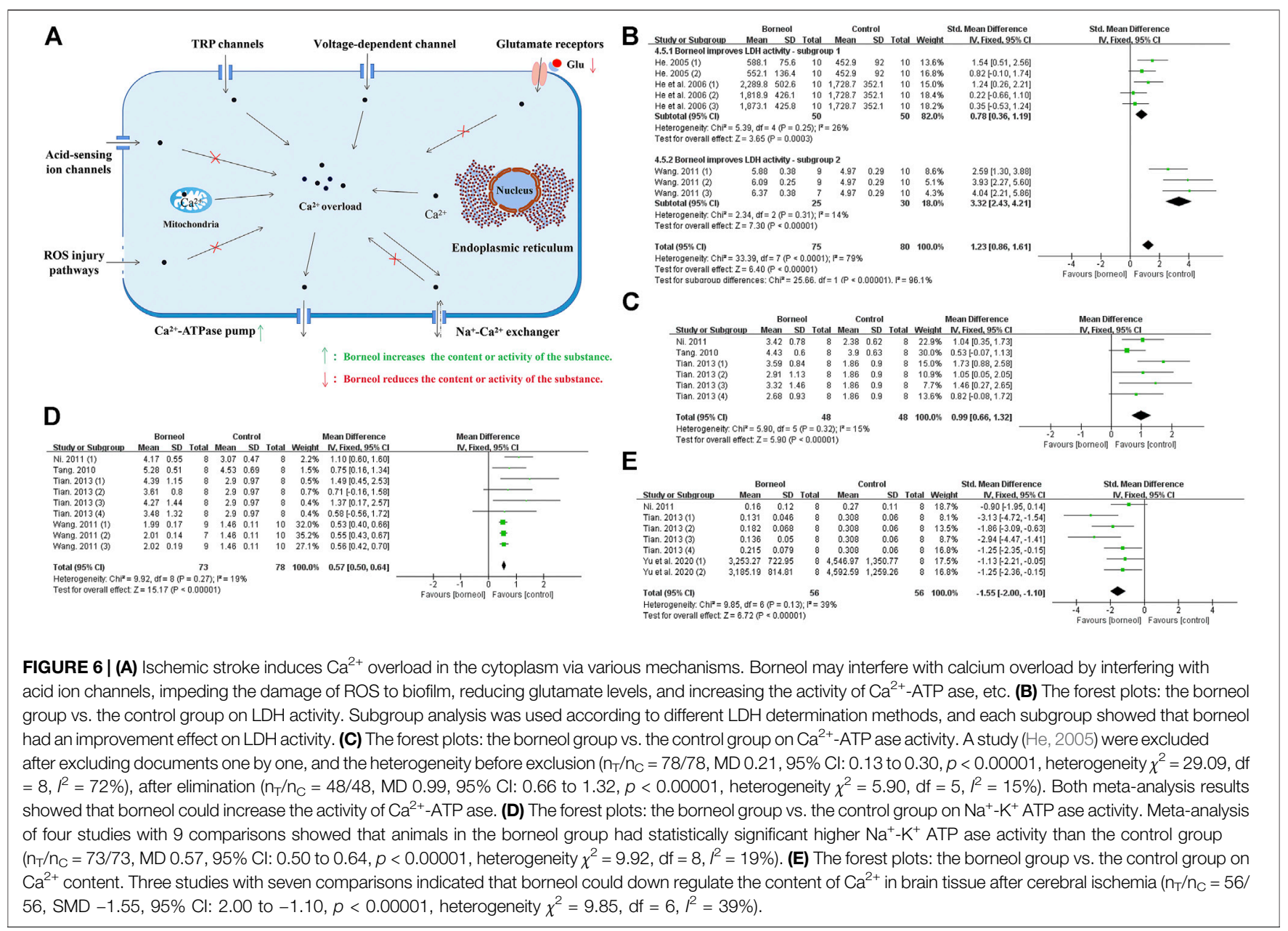

significantly decreased the concentration of $\mathrm{Ca}^{2+}$ in hippocampal and hypothalamic neurons after cerebral ischemia reperfusion (CIR) in rats (Yu et al., 2019a). Previous studies have found that borneol significantly increased the activity of lactate dehydrogenase (LDH) in ischemic brain tissue to inhibit the accumulation of lactic acid, which may interfere with $\mathrm{Ca}^{2+}$ entering cells through acid-sensitive ion channels (He, 2005; He et al., 2006; Wang, 2011). Besides, borneol reduces cytoplasmic $\mathrm{Na}^{+}$levels by increasing $\mathrm{Na}^{+}-\mathrm{K}^{+}$ATPase activity, which prevent $\mathrm{Ca}^{2+}$ from entering neurons via sodiumcalcium exchange $(\mathrm{He}, 2005)$. ROS increase cytoplasmic $\mathrm{Ca}^{2+}$ concentration by destroying the integrity of the biofilm, but borneol prevents the damage of biofilm by accelerating the scavenging of ROS. Glutamate mediates calcium influx in neurons by activating glutamate receptors, while borneol reduces the level of glutamate (Huang, 2018; Yu et al., 2019b). In addition, L-borneol and DL-borneol increased the expression and activity of $\mathrm{Ca}^{2+}-\mathrm{Mg}^{2+}$-ATPase, promoting the outflow of intracellular $\mathrm{Ca}^{2+}(\mathrm{Ni}, 2011$; Tian, 2013). The meta-analysis results also suggested that borneol could reduce the calcium content and increase the activities of $\mathrm{LDH}, \mathrm{Ca}^{2+}$-ATPase and $\mathrm{Na}^{+}-\mathrm{K}^{+}$-ATPase (Figure 6). Overall, these publications show that borneol can improve the concentration of intracellular $\mathrm{Ca}^{2+}$, block the pathological injury caused by $\mathrm{Ca}^{2+}$ overload, and interfere with the process of neuronal apoptosis in the cascade reaction. Mechanism of borneol inhibiting cytoplasmic $\mathrm{Ca}^{2+}$ overload and the meta-analysis results of related indicators are showed in Figure 6.

\section{Suppress Acute Hyperthermia}

During the acute stage of cerebral ischemia, fever increases the mortality and disability rates by aggravating neuronal damage (Wrotek et al., 2012). The leukocytes gather in the core area of cerebral infarction and release endogenous heat generators, and these cytokines raise the hypothalamic thermoregulation set point by triggering the release of arachidonic acid and activating cyclooxygenase to produce prostaglandins (Blatteis et al., 2005). Correlation analysis of clinical data and models of cerebral ischemia show that the harmful effects of fever after stroke are mediated by the increased excitotoxicity by glutamate, and the protective effect of hypothermia is also closely related to decreased glutamate release (Campos et al., 2012; Campos et al., 2013). Through feedback regulation, fever can further aggravate cerebral ischemic injury by promoting the secretion of excitatory neurotransmitters, increasing the production of ROS, accelerating the metabolic rate, and aggravating the 
degradation of neuronal cytoskeleton proteins (Ginsberg and Busto, 1998). Researchers accidentally found that both L-borneol and DL-borneol significantly inhibited the elevation of rats body temperature after pMCAO or tMCAO (Ni, 2011; Tian, 2013). We know that infection can also induce elevated body temperature in stroke patients. Studies have also shown that the three borneols reduced LPS-induced elevation of body temperature and reduced fever due to inflammation (Luo et al., 2016; Zou et al., 2017). These suggest that borneol can inhibit acute fever of ischemic stroke, and this process may involve the inhibition of glutamate excitotoxicity and reduction in the release of endogenous febrile factors.

\section{Mechanisms of Borneol Intervention in the Subacute Stage of Ischemic Stroke}

The physiological and pathological responses in the subacute stage of ischemic stroke (within $2-7^{\circ}$ days after the occurrence of human cerebral ischemia) play a key role in the transformation of brain injury in the infarcted area. Apoptosis and necrosis occur in the first few hours after ischemic stroke and reach a peak after $24 \mathrm{~h}$ (Wang et al., 2015b). Structural damage to the blood-brain barrier and the high expression of astrocyte aquaporins continue to aggravate brain edema and reach a peak 3-4 days after ischemia (Badaut et al., 2002; Badaut et al., 2007). Compared with the treatment of primary injury after arterial occlusion, secondary injury caused by inflammation may have a longer treatment time window. Intervening in the inflammatory response is an important means of treatment (Kawabori and Yenari, 2015). Therefore, the therapeutic use of borneol for blood-brain barrier damage, different types of cell death, and the inflammatory response in the subacute stage of ischemic stroke have important consequences for the treatment and rehabilitation of stroke patients.

\section{Intervention of Inflammatory Cytokine Expression}

Tumor necrosis factor alpha (TNF- $\alpha$ ), interleukin-1 $\beta$ (IL-1 $\beta$ ), and interleukin-6 (IL-6) are key inflammatory factors in cerebral ischemic injury. Nuclear factor kappa B (NF- $\mathrm{B}$ ) also plays an important role in the inflammatory process (Sun et al., 2014). All kinds of borneol significantly reduce TNF-a levels, while D-borneol and DL-borneol reduce IL-1 $\beta$ levels and DLborneol reduces IL-6 in cerebral ischemia/reperfusion models (Ni, 2011; Chang et al., 2017; Wen, 2017; Yu et al., 2017b; Dong et al, 2018). Myeloperoxidase (MPO) is a specific marker of neutrophils (Tu et al., 2010). D-borneol inhibits MPO activity, and inhibits neutrophil infiltration in the brain tissue of rats subjected to pBCO (Shao et al., 2018). Inhibition of cyclooxygenase-2 (COX-2) and 5-lipoxygenase (5-LOX) showed a neuroprotective effect in ischemic stroke rat model (Singh and Chopra, 2014). Cerebral ischemia/reperfusion induces the upregulation of COX-2 expression, resulting in the aggravation of the cerebral injury (Zhang et al., 2019). Cysteinyl leukotriene receptor 2 (CYSLTR2) is a subtype of cysteinyl leukotriene receptor, an inflammatory mediator (Zhang, 2013). Borneol inhibits the activities of 5-LOX and COX-2 of rats subjected to tMCAO and blocks CYSLTR2 expression in the hippocampus (Duan et al., 2012; Liu et al., 2018). As shown in Figure 7, the meta-analysis results suggested that borneol played an anti-inflammatory role by reducing the levels of TNF- $\alpha$, IL- $1 \beta$ and IL- 6 , and inhibiting the expression of 5-LOX and COX-2 in serum or brain tissue of cerebral ischemic animals. What's more, borneol inhibits proinflammatory factor release and cytoplasmic inhibitor of kappa $\mathrm{B}$ alpha (I $\mathrm{B} \mathrm{Ba})$ degradation, and blocks NF- $\kappa \mathrm{B}$ p65 nuclear translocation induced by OGD/R. Borneol also inhibits the release of TNF- $a$ and the expression of intercellular adhesion molecule-1 (ICAM1), reverses OGD/R-induced neuronal injury, nuclear pyknosis, ROS production, and the disappearance of mitochondrial membrane potential (Liu et al., 2011). Microglia-mediated neuroinflammation plays a crucial role in the pathophysiological process of multiple neurological disorders. Both LPS treatment and oxygen-glucose deprivation of neurons lead to the activation of microglia. Borneol has a direct inhibitory effect on the activation of microglia mediated by LPS in vitro. There was an increase in neuronal death after the addition of activated microglia culture supernatant, but the microglia culture medium treated with borneol reduced the toxicity of microglia to neurons with the decrease of TNF- $\alpha$, IL- $1 \beta$, and IL- 6 levels, and the increase of interleukin-10 (IL-10) levels (Wang et al., 2019). Taken together, these studies described above suggest that borneol protects against acute brain cell injury by attenuating inflammation. The mechanism of borneol attenuating inflammatory injury and the meta-analysis of related indicators are shown in Figure 7.

\section{Resistance to Damage of the Blood-Brain Barrier}

The blood-brain barrier (BBB) is a selective structural and functional barrier between the brain tissue and blood. The structural barrier is composed of vascular endothelial cells, pericytes, basal lamina, and astroglial terminal feet (Ueno, 2009). The functional barrier depends on P-glycoprotein (P-gp), which can expel specific substances that enter the endothelial cells back into the bloodstream (Ueno et al., 2010). The protective effect of borneol on the BBB is achieved by structurally up-regulating the expression of tight junction proteins in vascular endothelial cells, inhibiting structural destruction of the BBB mediated by metalloproteinases, and functionally inhibiting the efflux of P-gp on neuroprotective drugs.

\section{Direct Protective Effect on $B B B$}

The meta-analysis results of several studies confirmed that borneol could reduce the infiltration of evans blue (EB) into the brain and decrease the brain water content after cerebral ischemia (Jia, 2014; Liu et al., 2017; Dong et al., 2018; Wang, 2011; Yao et al., 2011) (Figure 8). The mechanism of borneol inhibits $\mathrm{BBB}$ structural damage may involve the downregulation of matrix metallopeptidase 2 (MMP2) and matrix metallopeptidase 9 (MMP9), and the upregulation of TIMP metallopeptidase inhibitor 1 (TIMP1). MMP9 and MMP2 are proteases that promote the opening of the $\mathrm{BBB}$ and the formation of brain edema by degrading the basal lamina of cerebral vessels. TIMP1 antagonizes MMP2 and MMP9 activity. DL-borneol increases 


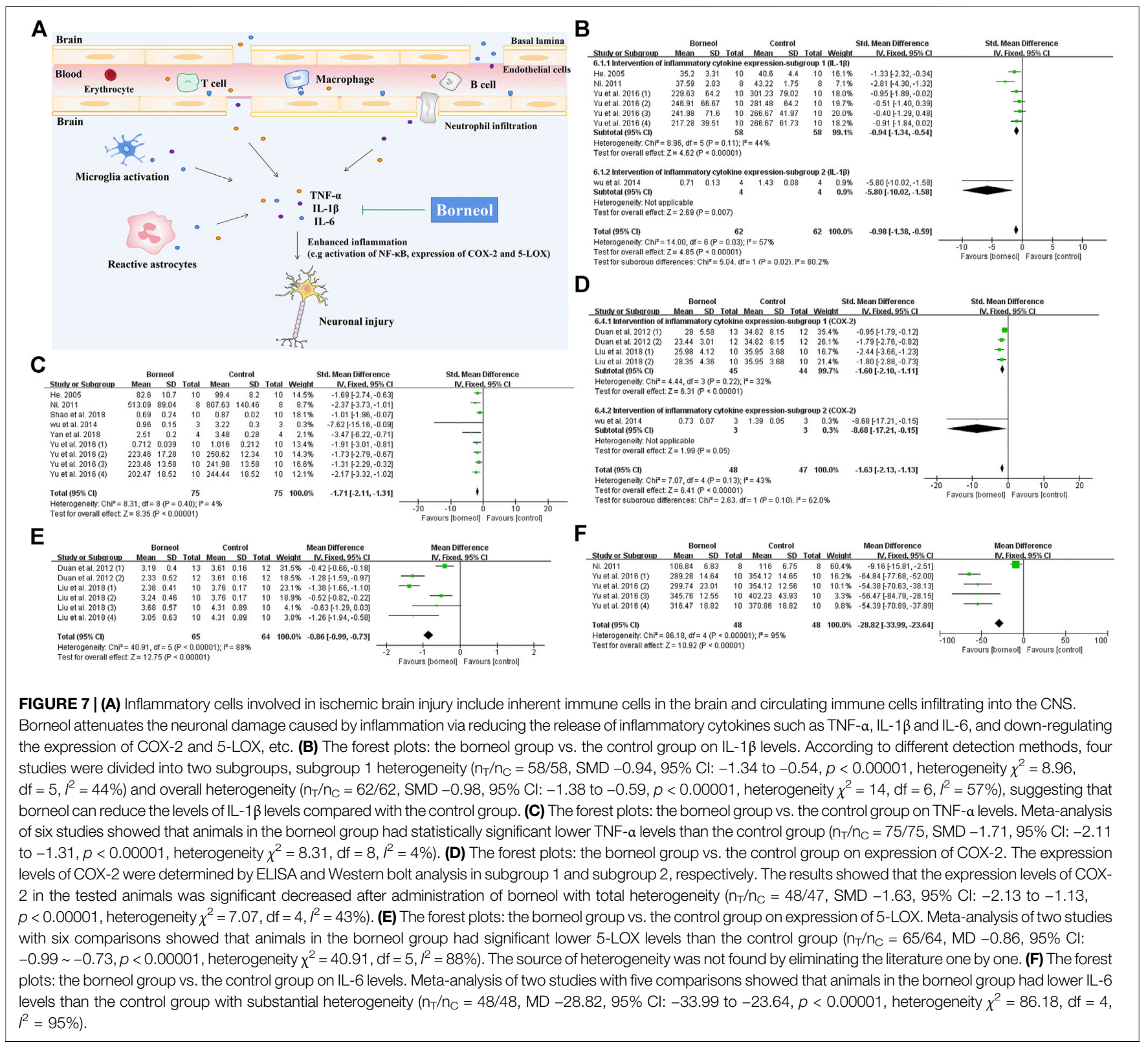

TIMP1 mRNA expression in rats subjected to tMCAO (Liu et al., 2007). D-borneol downregulates MMP2 and MMP9 expression in rats subjected to pMCAO, and DL-borneol inhibits MMP9 mRNA and protein in a tMCAO rat model (Liu et al., 2009; Wang et al., 2018).

In addition to the effects on metalloproteinases, borneol may also downregulate the expression of ICAM1 and lymphocyte function-associated antigen 1 (LFA-1). ICAM1 is a transmembrane protein expressed on vascular endothelial cells that binds to LFA-1 in inflammatory site. The combination of LFA1 and ICAM1 facilitate leukocyte adherence to, or passage through vascular endothelial cells to reach the focal area of ischemia. Borneol inhibits the adhesion and penetration of leukocytes to endothelial cells by inhibiting the expression of ICAM1 and LFA-1 (Liu et al., 2009; Kong et al., 2013; Liu et al., 2011).
Borneol upregulates claudin 5 (CLDN5) and tight junction protein 1 (TJP1) expression. CLDN5 and TJP1 are key components of tight junctions between cerebral microvascular endothelial cells, and their expression is significantly decreased in ischemic brain tissue (Li et al., 2017). Previous studies have confirmed that D-borneol upregulates CLDN5 and TJP1 expression in tMCAO model rats and brain microvascular endothelial cells with OGD /R treatment (Xu and Zhang, 2015; Xu and Zhang et al., 2016). L-borneol and DL-borneol increased the expression of CLDN5 in an animal model of permanent cerebral ischemia (Xu et al., 2016; Wen, 2017; Dong et al., 2018; Wang et al., 2018).

In addition, aquaporin 4 (AQP4) is concentrated at the foot processes of astrocytes. The outer surface of blood vessels is almost surrounded ( $85 \%)$ by the end feet of glial cells. Thus, increased expression of this protein can induce brain edema $(\mathrm{Hu}$ 


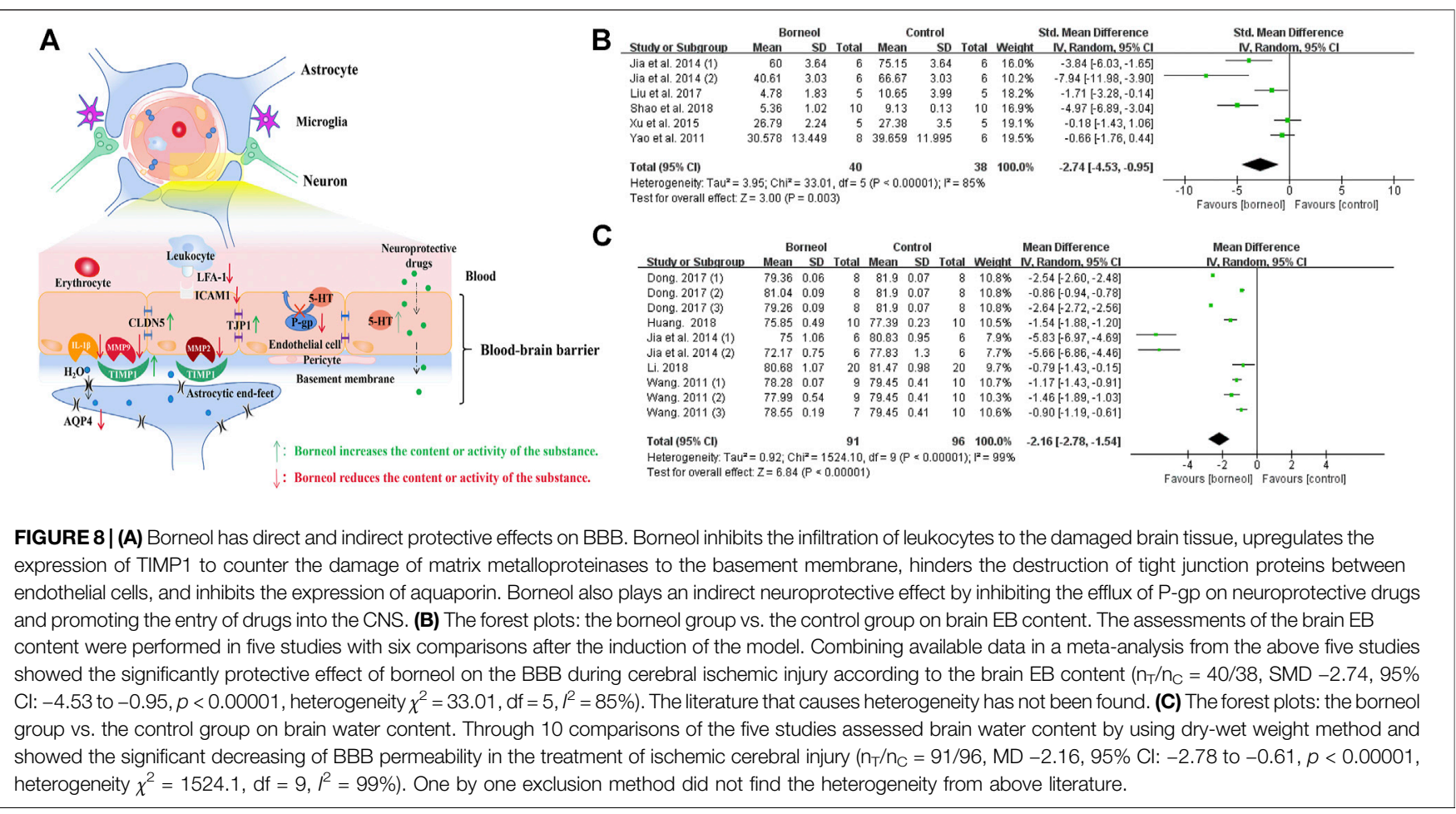

et al., 2012). Borneol inhibits brain edema after cerebral ischemia, which may be related to the downregulation of AQP4 mRNA expression (Liu et al., 2009).

\section{Indirect Protective Effect on $B B B$}

The anatomy of the $\mathrm{BBB}$ protects the central nervous system (CNS) from toxins and variations in blood composition, and maintains the consistency of the brain's micro-environment (Abbott and Friedman, 2012). Although considerable advancements have been made in drug delivery to the CNS, the clinical application of CNS drugs is still limited by their poor bioavailability due to the BBB (Denora et al., 2009). Borneol promotes the penetration and accumulation of neuroprotective drugs such as gastrodin, puerarin, kaempferol, and nimodipine, increases the bioavailability of these drugs, thereby exerting an indirect neuroprotective effect (Cai et al., 2008; Gao et al., 2010; Wu et al., 2014a; Zhang et al., 2015). The mechanism of this effect is closely related to the regulation of P-gp.

One study showed that P-gp was expressed 30 min after focal cerebral ischemia in rats and lasted for $24 \mathrm{~h}$ (Xing et al., 2007). Borneol downregulates the expression of P-gp both physiological and pathological conditions. L-borneol inhibits P-gp expression in rats subjected to tMCAO (Yu et al., 2011; Tian, 2013). P-gp, which is encoded by $M d r 1 a$, is expressed on the luminal membrane of brain microvascular endothelial cell (BMEC) and confers multidrug resistance to various chemotherapeutic agents (Chen et al., 2003). In an in vitro BBB model composed of rat brain BMECs and astrocytes, L-borneol downregulated the efflux function of P-gp by inhibiting the expression of $m d r l a$ mRNA and P-gp, and this process was related to the transient activation of NF- $\kappa B$ (Fan et al., 2015).
Borneol also affects 5-HT, a known BBB neurotransmitter (Ke et al., 2000). Borneol can mediate BBB opening by increasing 5HT in the hypothalamus of rats (Hui et al., 2009). Since P-gp is a lipophilic protein (Abbott et al., 2010), borneol easily binds to $\mathrm{P}$-gp after passing through the BBB and inhibits the binding of 5HT to $\mathrm{P}$-gp, which results in a reduction in $\mathrm{P}$-gp-mediated efflux of 5-HT and a consequent increase in 5-HT in cerebral vascular endothelial cells (Yuan et al., 2006). The mechanism of borneol protecting $\mathrm{BBB}$ and results of meta-analysis of related indicators are shown in Figure 8.

\section{Regulation of Various Forms of Cell Death}

Previous studies applied TTC staining to evaluate cerebral infarction area, showing that borneol had significant differences in alleviating cerebral infarction (Wen, 2017; Dong et al., 2018). The cytotoxic effects of excitatory amino acid toxicity, oxidative stress, and ROS damage in the acute stage of cerebral ischemia lead to rapid disintegration and necrosis of neurons. Delayed neuronal injury after several days of ischemia exhibits characteristics of apoptosis. Cellular injury in the central necrotic area of the cerebral infarction is irreversible, but the peripheral ischemic penumbra is salvageable (Guo, 2000). BCL2 apoptosis regulator (BCL2) and BCL2 associated X, apoptosis regulator $(\mathrm{BAX})$ are typical BCL-2 family apoptosis-inhibiting protein and apoptosis-inducing protein, respectively. Regulating the balance between BAX and BCL2 can hinder the expansion of infarct area (Xu et al., 2016). Previous reports demonstrated that D-borneol and L-borneol reduced the BAX/BCL2 ratio in rats subjected to pMCAO and DL-borneol reduced the BAX/BCL-2 ratio in both pMCAO and tMCAO models (Huang, 2018; Wen, 2017). Caspase-3 (CASP3) induces neuronal apoptosis in 


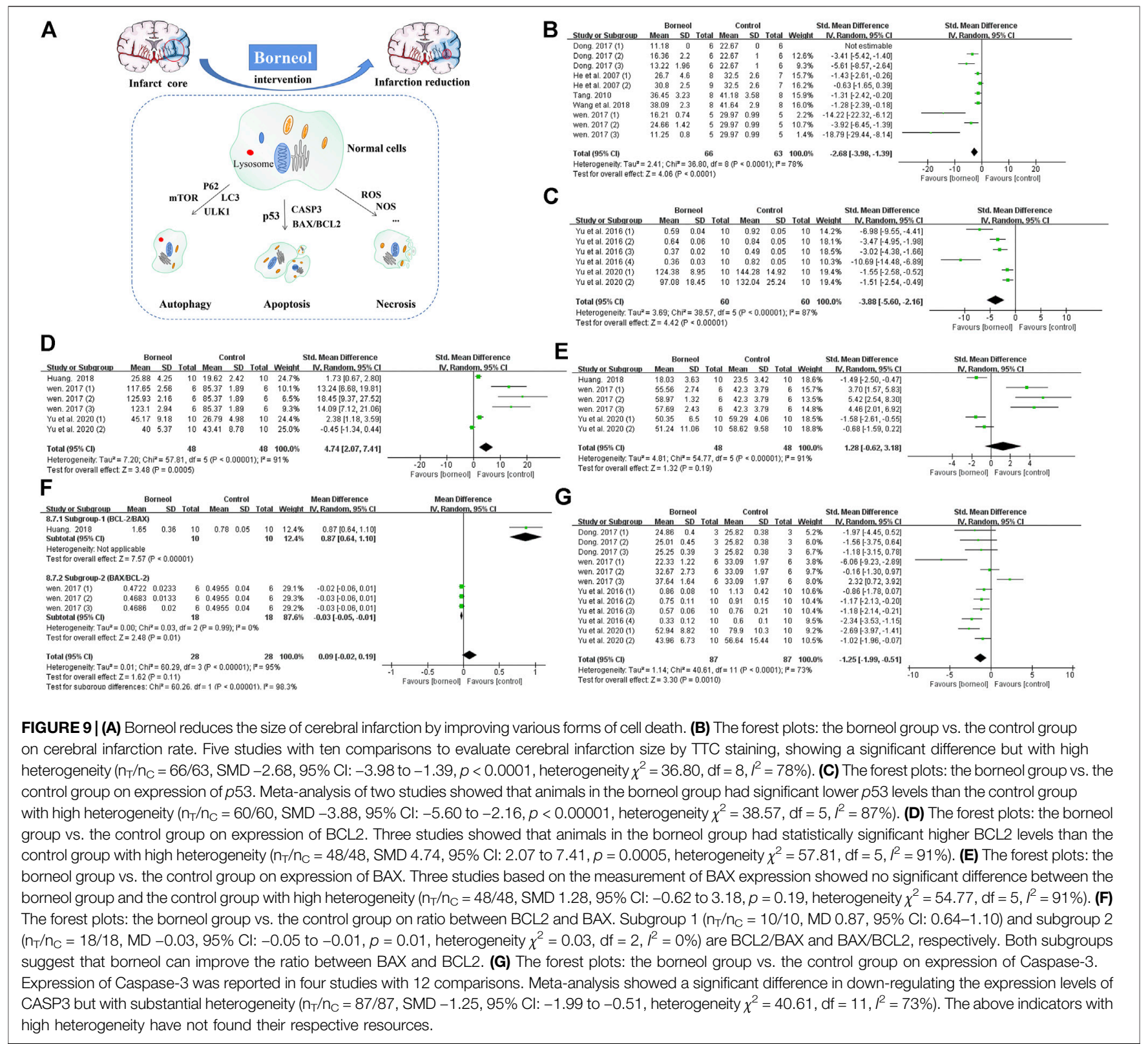

ischemic stroke, and which is interrupted by L-borneol and D-borneol after cerebral ischemia/reperfusion (Wen, 2017; Yu et al., 2020). Tumor protein 53 (p53) is mainly expressed in the mitochondria and contributes to cell apoptosis (Wang et al., 2017b). Borneol reduces p53 expression in the cortex and striatum (Yu et al., 2020). Meta-analysis results also suggested that borneol had anti-apoptotic effects by down-regulating the expression levels of p53 and caspase-3, and regulating the ratio of BAX and BCL-2 (Figure 9). Furthermore, borneol enhances the neuron protective autophagy in the cortex and striatum by modulating beclin1, mTOR, and LC3II/I (Yu et al., 2017c; Yu et al., 2020). L-camphor is one of the metabolic products of borneol in the body (Jiang et al., 2008). Several studies have demonstrated that L-camphor upregulates cell adhesion molecule 2 (CADM2) expression to promote neurite outgrowth by targeting microRNA-125a and microRNA-140 and upregulates heterogeneous nuclear ribonucleoprotein A1 (HNRNPA1) expression to promote the expression of stress granules. At the same time, L-camphor can improve apoptosis and autophagy by regulating the autophagy-related proteins, p62 and LC3, and apoptosis-related proteins BCL-XL and CASP3 (Li, 2016; Liu, 2017; Ren, 2018). The mechanism of borneol intervention in various forms of neuronal death and related meta-analysis results are shown in Figure 9.

\section{Mechanism of Borneol Intervention in the Late Stage of Ischemic Stroke}

In the late stage of ischemic stroke (one week after the occurrence of cerebral ischemia in humans), reactive glial cell hyperplasia 
and glial scar formation limit the expansion of brain damage and separate the necrotic infarct core from the surrounding normal tissues (Sims and Yew, 2017). The reconstruction of the glial cell groups, the neovascularization of the blood vessels, and the regeneration of the nerve myelin sheath play leading roles in the repair process at this stage. Borneol helps the body recover to a healthy physiological state more quickly by promoting angiogenesis and accelerating the repair of damaged neurons in the late stage of stroke.

\section{Promoting the Growth and Repair of Neurons and Angiogenesis}

Animal experiments confirmed that borneol improved the neurological function score and reduced the cerebral infarction area (Wu et al., 2014b; Dong et al., 2018). Immunofluorescence staining showed that borneol also lowered neuronal mortality and promoted neurogenesis (Zhang et al., 2017b; Yu et al., 2020). Tanshinol borneol ester (DBZ) is a novel synthetic compound derived from Dantonic ${ }^{\circledR}$. DBZ plays a role in inducing angiogenesis in both in vivo and in vitro experiments (Liao et al., 2019). Borneol promotes neurogenesis and angiogenesis in the brain, possibly through the upregulation of endogenous neurotrophic factors and regulation of the $\mathrm{Wnt} / \beta$-catenin pathway.

\section{Increasing Endogenous Neurotrophic Factors}

Ischemic brain injury destroys nerves and cerebral vessels and induces compensatory neonatal reactions in brain tissue, including nerve regeneration and vascular remodeling. Borneol regulates brain derived neurotrophic factor (BDNF), nerve growth factor (NGF), vascular endothelial growth factor (VEGF), fibroblast growth factor 2 (FGF2/bFGF), and glial cell derived neurotrophic factor (GDNF), and promotes repair of nerves as well as remodeling of blood vessels by activating the $\mathrm{Wnt} / \beta$-catenin pathway.

In a tMCAO rodent model, DL-borneol significantly decreased VEGF mRNA on the first day after cerebral ischemia but increased VEGF mRNA levels on the second and third days (Hu et al., 2005; Ni, 2011). Three kinds of borneol can upregulate the expression of VEGF in a pMCAO rat model after ischemia (Dong et al., 2018). Under physiological conditions, endothelial cells express approximately 10 times less fms related receptor tyrosine kinase 1 (FLT1/VEGFR1) than kinase insert domain receptor (KDR/VEGFR2) (DeVal and Black, 2009). Binding of VEGF to VEGFR2 activates intracellular tyrosine kinases and multiple downstream signals that induce angiogenesis. The inhibition of VEGFR2 signaling disturbs endothelial cell proliferation after stroke (Shimotake et al., 2010). On the contrary, VEGFR1 negatively regulates cell proliferation and reduces angiogenesis (Mendes et al., 2018). Increasing evidence suggests that cerebral ischemia upregulates VEGFR1 expression, which positively correlates with the degree of damage (Yoo et al., 2010; Causey et al., 2012). In a BMECs model treated with OGD, borneol increased the expression of VEGF, reduced the expression of VEGFR1, and tended to increase the expression of VEGFR2 (Yu et al., 2019a). The latest research confirms that L-borneol can promote angiogenesis coupled neurogenesis by regulating Ang1-VEGFBDNF to play a neuroprotective effect (Ma et al., 2021). In a tMCAO rat model, borneol significantly enhanced the expression of NGF, BDNF, GDNF, and VEGF mRNA after $48^{\circ} \mathrm{h}$ of reperfusion. Borneol not only increased the expression of $N G F, \quad G D N F$, and VEGF mRNA but also significantly increased the level of $b F G F$ mRNA after $72 \mathrm{~h}$ of reperfusion (Hu, 2004).

\section{Regulation of the Wnt/ $\beta$-Catenin Pathway}

The $\mathrm{Wnt} / \beta$-catenin pathway regulates neuronal differentiation and microangiogenesis, and this pathway is crucial for endothelial cell maintenance of the BBB homeostasis and normal neural function. The $\mathrm{Wnt} / \beta$-catenin pathway participates in the proliferation and differentiation of neural stem cells, the formation of axons, the occurrence of cortical patterns, vascular regeneration and remodeling in the nervous system, and the formation of the BBB. Wnt family member $3 \mathrm{~A}$ (WNT3A), $\beta$-catenin, disheveled (Dsh), and lymphoid enhancer binding factor 1 (LEF1) are positive regulatory molecules of the $\mathrm{Wnt} / \beta$-catenin pathway, while glycogen synthase kinase 3 beta (GSK3B) and APC regulator of WNT signaling pathway (APC) are negative regulators of this pathway (Wen et al., 2016). Three kinds of borneol activate the Wnt/ $\beta$-catenin signal pathway by regulating the expression of positive and negative regulatory molecules in rats subjected to pMCAO, performing the neuroprotective effect of CNS (Wen, 2017). In another study, the combination of borneol, astragaloside IV, and Panax notoginseng saponins promotes the proliferation of neurons and repairs damaged neurons, enhancing the resistance of rats to cerebral ischemia/reperfusion injury, which is also associated with the regulation of the Wnt/ $\beta$-catenin signaling pathway (Yang et al., 2019).

\section{DISCUSSION}

\section{Summary of Results}

Ischemic brain injury is an extremely complex process, but the neuroprotective effect of many drugs is more likely to play a single role in the ischemic cascade reaction. In summary, borneol has protective effects at all three stages of cerebral ischemic injury. In the acute stage of cerebral ischemia, borneol improves cerebral blood flow by relaxing blood vessels and eliciting anti-thrombotic effects, inhibits neuronal excitotoxicity by reducing Glu levels and activating $\mathrm{GABA}_{\mathrm{A}}$ receptors, improves the ability of the body to resist ROS injury by increasing the activity of antioxidant enzymes, regulates the activity of different types of NOS and antagonizes the neurotoxic effect of NO, reduces the content of intracellular $\mathrm{Ca}^{2+}$, interferes with the process of neuron death, and antagonizes the symptoms of acutely elevated body temperature. In the subacute phase of ischemia stroke, borneol interferes with the expression of inflammatory cytokines, the destruction of $\mathrm{BBB}$, and multiple forms of neuron death. In the late stage of brain injury, borneol promotes neuronal repair and angiogenesis. Furthermore, borneol mobilizes endogenous nutritional factors to hasten self-repair of the body, avoiding 

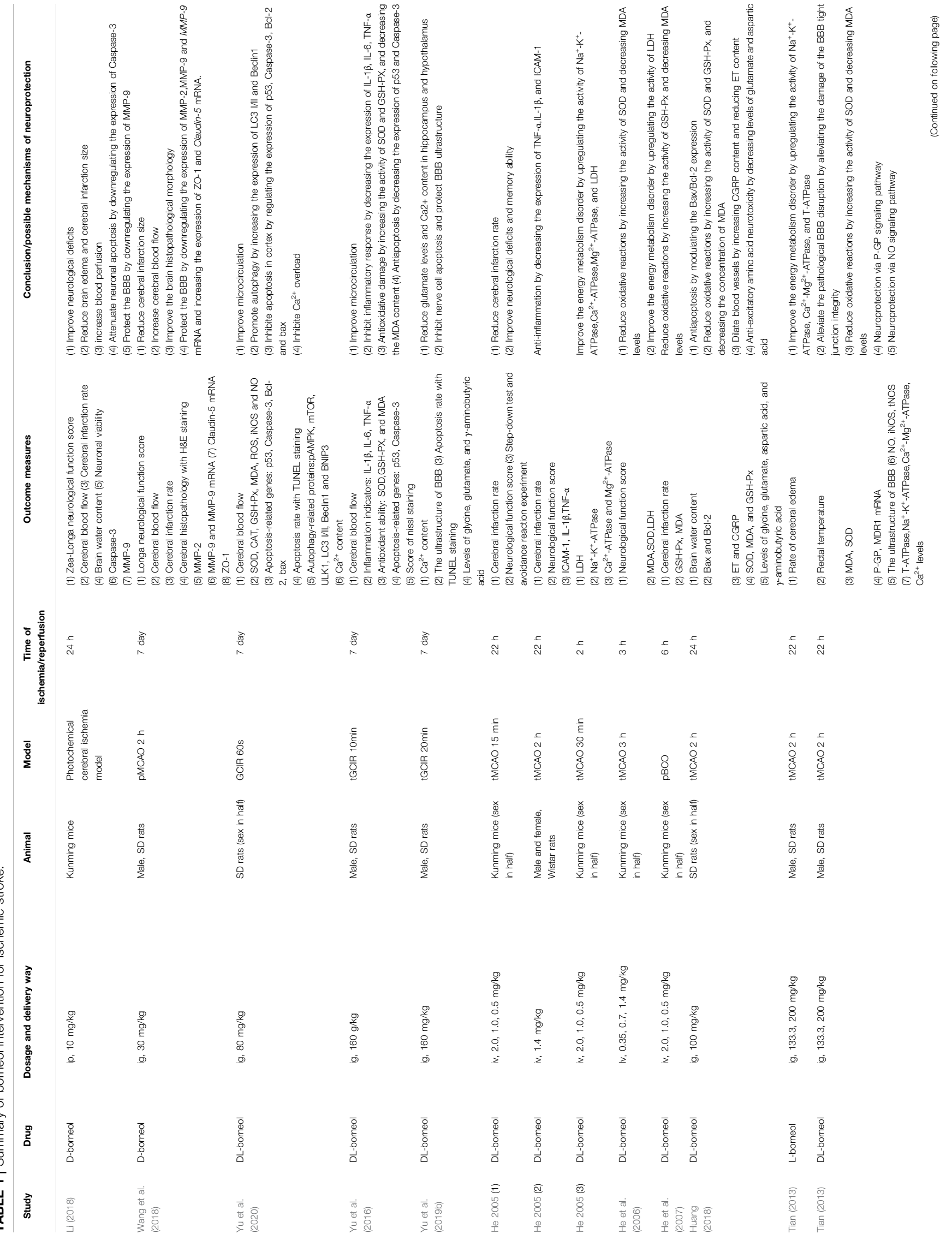

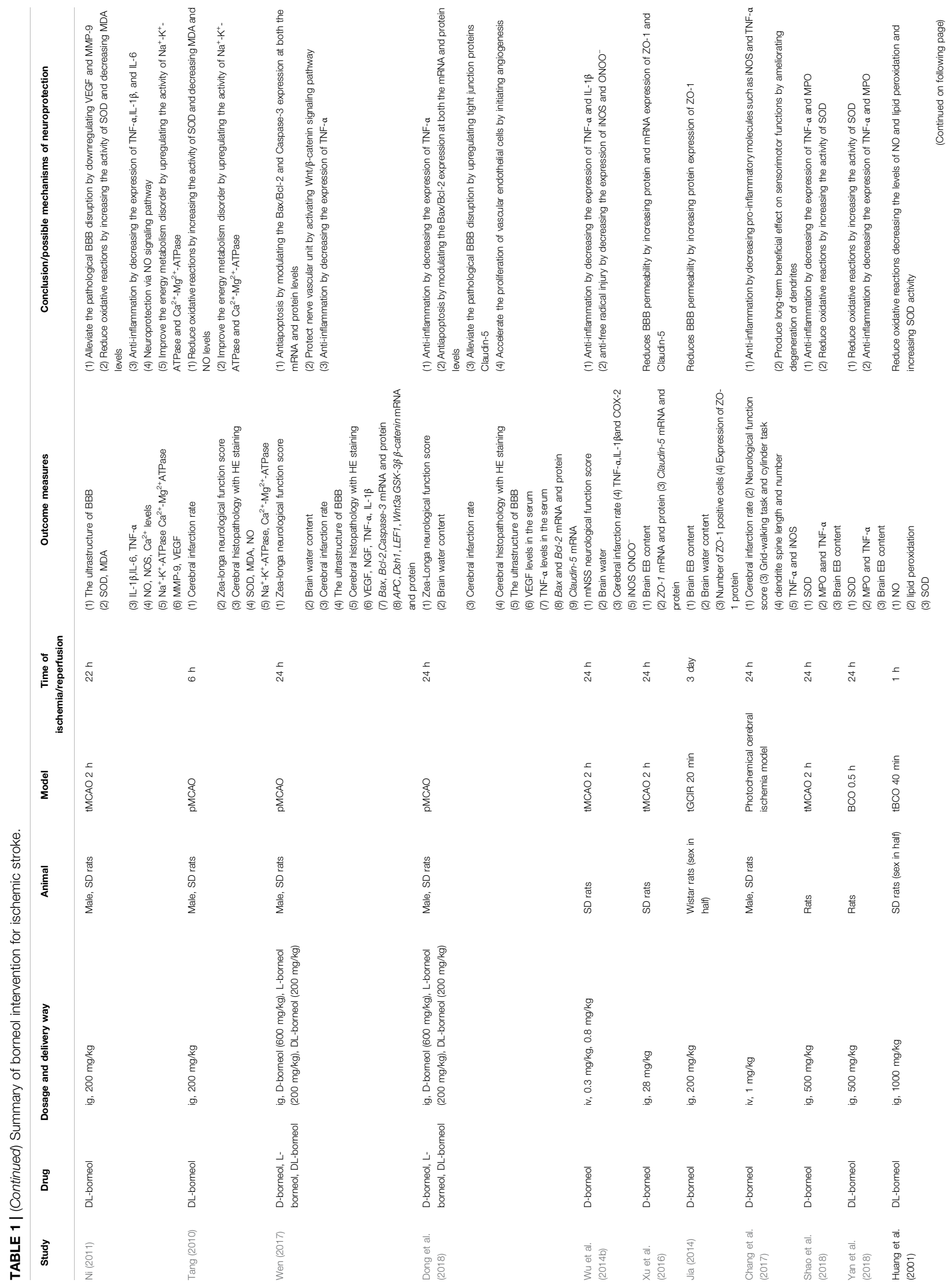
the defect of single-target therapeutic agents. Moreover, borneol can promote other drugs to pass through the $\mathrm{BBB}$ to enhance their therapeutic effects and play a synergistic neuroprotective effect. Hence, brneol can interfere with neuronal injury after cerebral ischemia through multiple channels.

\section{Implications}

Borneol is a naturally occurring product in a class of "orificeopening" agents used in TCM for resuscitative purpose, and is widely used as an upper ushering drug for various brain diseases in many Chinese herbal formulae (Zhang et al., 2017a; Chen et al., 2019). This is consistent with the findings of many scholars that borneol has a beneficial effect on increasing the bioavailability, tissue distribution, and blood concentration of other drugs, and making other drugs transport through BBB easier (Xiao et al., 2007; Cai et al., 2008; Lu et al., 2011).

Heat-clearing is a traditional effect of borneol as a resuscitation-inducing aromatic medicine (Zou et al., 2017; Wang and Wang, 2018). Some researchers found that oral administration of borneol had a certain improvement effect on fever in the acute phase of stroke, which is consistent with the understanding of "inducing resuscitation with drugs of pungent flavor and cool naturenature" of borneol in TCM clinical practice. But the time-effect, dose-effect relationships, and mechanisms of borneol inhibiting stroke hyperpyrexia deserve a more thorough study.

Oral administration is the most common route in the clinical use of borneol. Borneol is absorbed rapidly into the brain and has the same concentration in the brain as in the blood within $5 \mathrm{~min}$ of oral administration (Pan et al., 2014). In mice, a single oral dose of borneol accumulates in organs in this order: lung $<$ muscle $<$ spleen $<$ heart $<$ kidney $<$ brain $<$ liver (Huang et al., 2009). The distribution of borneol in the brain also shows regional specificity, with the highest concentration in the cortex, moderate concentrations in the hippocampus and hypothalamus, and lowest concentration in the striatum (Yu et al., 2013). Besides, the biphasic half-life and elimination of D-borneol and DLborneol in the plasma were $0.7-8.5 \mathrm{~h}$ and $0.8-8.0 \mathrm{~h}$, respectively, (Cheng et al., 2013). Another study found that $10 \mathrm{~h}$ after a single dose of $2 \mathrm{~g} \mathrm{~L}$-borneol, about $81 \%$ of borneol is excreted in the form of glucuronic acid binding in urine (Bhatia et al., 2008). These studies suggest that while borneol is easily absorbed, it does not easily accumulate in the body.

\section{Outlooks}

In Table 1, we found that borneol administration in ischemic stroke was mainly oral, with some instances of intravenous administration being recorded. However, it has been reported that intranasal administration of borneol had rapid absorption into the blood and brain compared with oral administration while having similar bioavailability compared to intravenous administration (Zhao et al., 2012). Therefore, it is necessary to pay more attention to the nasal administration of borneol in the future research of brain diseases.

It is undeniable that only few patients receive thrombolysis within 6-8 $\mathrm{h}$ in the treatment of cerebral ischemia, which results in most patients developing permanent cerebral ischemia 
TABLE 2 | Quality assessment of included studies.

\begin{tabular}{|c|c|c|c|c|c|c|c|c|}
\hline $\begin{array}{l}\text { Study } \\
\text { years }\end{array}$ & A & B & C & D & $\mathbf{E}$ & $\mathbf{F}$ & G & Total \\
\hline Li (2018) & + & + & + & & + & + & + & 6 \\
\hline Wang et al. (2018) & + & + & & & + & & + & 4 \\
\hline Yu et al. (2020) & + & + & & & + & + & + & 5 \\
\hline Yu et al. (2016) & + & + & & & + & + & + & 5 \\
\hline Yu et al. (2019a) & + & + & & & + & & + & 4 \\
\hline $\mathrm{He}(2005)$ & + & + & & & + & & + & 4 \\
\hline He et al. (2006) & + & + & & & + & + & + & 5 \\
\hline He et al. (2007) & + & + & & & + & + & + & 5 \\
\hline Huang (2018) & + & + & & & + & + & + & 5 \\
\hline Tian (2013) & + & + & & & + & + & + & 5 \\
\hline $\mathrm{Ni}(2011)$ & + & + & & & + & + & + & 5 \\
\hline Tang (2010) & + & + & & & + & + & + & 5 \\
\hline Wen (2017) & + & + & & & + & + & + & 5 \\
\hline Dong et al. (2018) & + & + & & & + & + & + & 5 \\
\hline Wu et al. (2014) & + & + & + & & + & + & + & 6 \\
\hline Xu et al. (2016) & + & + & & & + & & + & 4 \\
\hline Jia (2014) & + & + & & & + & + & + & 5 \\
\hline Chang et al. (2017) & + & + & + & & + & + & + & 6 \\
\hline Shao et al. (2018) & + & + & & & + & & + & 4 \\
\hline Yan et al. (2018) & + & + & & & + & & + & 4 \\
\hline Huang et al. (2001) & + & + & & & & & + & 3 \\
\hline Wang (2011) & + & + & & & + & + & + & 5 \\
\hline Duan et al. (2012) & + & + & & & + & & + & 4 \\
\hline Liu et al. (2018) & + & + & & & & & + & 3 \\
\hline Liu et al. (2007) & + & + & & & + & & + & 4 \\
\hline Yao et al. (2011) & + & + & & & + & & + & 4 \\
\hline
\end{tabular}

$A$, peer-reviewed publication; $B$, random allocation; $C$, blinded conduct of the Experiments; $D$, blinded assessment of outcome; $E$, use of anesthetic without significant neuroprotection; F, compliance with animal welfare regulations; $G$, detailed description of animals and models.

(Chalela et al., 2007). Regrettably, most experimental studies being focused on transient ischemic stroke (Mcbride and Zhang, 2017; Ma et al., 2020). This phenomenon can also be seen in Table 1. Thus, the comparative study between permanent cerebral ischemia model and ischemia-reperfusion model has farreaching clinical value for the differential medication of borneol in the treatment of stroke.

As we all know, there are pharmacodynamic differences between the compounds which are optically active isomers (Wermuth, 2015). Borneol is a bicyclic compound with three chiral carbon atoms and several optical isomers. L-borneol and D-borneol are a pair of optical isomers, and their neuroprotective effects in ischemic injury may be different. Consequently, the comparison of pharmacodynamic differences of different borneols in the intervention of ischemic stroke should be one of the focuses of later work. Besides, it is worth noting that there are many commercial species or isomers of borneol, and the botanical taxonomic names used by researchers are not the same, which makes it difficult to compare the experimental results. Therefore, it is also necessary to clarify the botanical source, spatial structure and drug purity of borneol in future experimental studies.

\section{Limitations}

The mechanisms of ischemic brain damage in humans and experimental animals cannot be equal because of species differences. This paper is based on the injury mechanism in humans at different periods (acute stage, subacute stage, and late stage) as a clue to sort out the neuroprotective effect of borneol in experimental models. Therefore, whether borneol can exert the same protective mechanism in clinical practice requires further study.

Some meta-analysis results have high heterogeneity due to experimental animals, the types and doses of borneol, animal models, and other factors. Therefore, exploring the reason that causes substantial heterogeneity and conducting more detailed data analysis will be issues that need to be addressed by relevant practitioners later.

The quality scores for the included studies shown in Table 2. The average quality score of in vivo animal studies for meta-analysis was 4.6. Twenty-six studies were peerreviewed publications, among them, nine studies were published master's thesis or PhD thesis. Twenty-six studies declared the random allocation. Three studies reported the masked conduct of experiments. None of the included studies described the blinded assessments of outcome. Twenty-four studies described the use of anesthetic without significant intrinsic neuroprotective activity. Fifteen studies stated they compliance with animal welfare regulations. Twenty-six studies described the experimental animals and the model preparation process in comparatively detail. According to the above quality assessment results, we call for more high-quality studies to confirm the neuroprotective effect and mechanism of borneol in the future.

\section{CONCLUSION}

Although borneol is rarely used alone in the clinical treatment of brain diseases, it cannot be ignored that borneol exerts a significant neuroprotective effect even when used alone in vivo and in vitro studies. The meta-analysis results of various animal experiments further indicated that borneol has an intervention effect in energy metabolism, inflammatory reaction, apoptosis, and necrosis as well as other processes of cerebral ischemic cascade reaction. This possible explanation for the benefits and the success of borneol in preventing enlargement of infarction and improving neurological function score. Overall, the previous review excessively focused on the regulation of borneol on $\mathrm{BBB}$ and ignored its overall effect on the treatment of cerebral ischemia. But this review comprehensively highlights the potential application of borneol as a neuroprotective agent against cerebral ischemia. We strongly believe that the research field of borneol antiischemic stroke is still promising.

\section{AUTHOR CONTRIBUTIONS}

YL, MR, JW (3rd author), QX, HL, and JL of our team are responsible for collecting relevant documents, $\mathrm{YL}$ is responsible for writing the first draft of the paper, JW (9th author), RM and $\mathrm{HC}$ are responsible for reviewing the manuscript, and YL is responsible for submitting the final manuscript. 


\section{FUNDING}

This work is financially supported by grants from National Natural Science Foundation of China (81873023 and 81473371).

\section{REFERENCES}

Abbott, N. J., and Friedman, A. (2012). Overview and Introduction: the BloodBrain Barrier in Health and Disease. Epilepsia. 53, 1-6. doi:10.1111/j.1528-1167. 2012.03696.x

Abbott, N. J., Patabendige, A. A. K., Dolman, D. E. M., Yusof, S. R., and Begley, D. J. (2010). Structure and Function of the Blood-Brain Barrier. Neurobiol. Dis. 37 (1), 13-25. doi:10.1016/j.nbd.2009.07.030

Badaut, J., Lasbennes, F., Magistretti, P. J., and Regli, L. (2002). Aquaporins in Brain: Distribution, Physiology, and Pathophysiology. J. Cereb. Blood Flow Metab. 22, 367-378. doi:10.1097/00004647-200204000-00001

Badaut, J., Ashwal, S., Tone, B., Regli, L., Tian, H. R., and Obenaus, A. (2007). Temporal and Regional Evolution of Aquaporin-4 Expression and Magnetic Resonance Imaging in a Rat Pup Model of Neonatal Stroke. Pediatr. Res. 62 (3), 248-254. doi:10.1203/PDR.0b013e3180db291b

Bhatia, S. P., McGinty, D., Letizia, C. S., and Api, A. M. (2008). Fragrance Material Review on L-Borneol. Food Chem. Toxicol. 46 (Suppl. 1111), S81-S84. doi:10.1016/j.fct.2008.06.054

Blatteis, C. M., Li, S., Li, Z., Feleder, C., and Perlik, V. (2005). Cytokines, PGE2 and Endotoxic Fever: a Re-assessment. Prostaglandins Other Lipid Mediat. 76 (1), 1-18. doi:10.1016/j.prostaglandins.2005.01.001

Cai, Z., Hou, S., Li, Y., Zhao, B., Yang, Z., Xu, S., et al. (2008). Effect of Borneol on the Distribution of Gastrodin to the Brain in Mice via Oral Administration. J. Drug Target. 16 (2), 178-184. doi:10.1080/10611860701794395

Campos, F., Pérez-Mato, M., Agulla, J., Blanco, M., Barral, D., Almeida, Á., et al. (2012). Glutamate Excitoxicity Is the Key Molecular Mechanism Which Is Influenced by Body Temperature during the Acute Phase of Brain Stroke. Plos One. 7 (8), e44191. doi:10.1371/journal.pone.0044191

Campos, F., Sobrino, T., Vieites-Prado, A., Pérez-Mato, M., Rodríguez-Yáñez, M., Blanco, M., et al. (2013). Hyperthermia in Human Ischemic and Hemorrhagic Stroke: Similar Outcome, Different Mechanisms. PLoS ONE. 8, e78429. doi:10. 1371/journal.pone.0078429

Causey, M. W., Salgar, S., Singh, N., Martin, M., and Stallings, J. D. (2012). Valproic Acid Reversed Pathologic Endothelial Cell Gene Expression Profile Associated with Ischemia-Reperfusion Injury in a Swine Hemorrhagic Shock Model. J. Vasc. Surg. 55 (4), 1096-1103. doi:10. 1016/j.jvs.2011.08.060

Chai, L. j., Xu, Y., Huang, J. Y., Li, R. L., Guo, H., and Wang, S. X. (2019). Xingnaojing Injection Components Promote Glutamate Clearance in Hypoxia/ reoxygenation Astrocytes and Improve Cell Viability in Hypoxia Neurons. Tianjin J. Traditional Chin. Med. 36 (3), 283-287. doi:10.11656/j.issn.16721519.2019.03.19

Chalela, J. A., Kidwell, C. S., Nentwich, L. M., Luby, M., Butman, J. A., Demchuk, A. M., et al. (2007). Magnetic Resonance Imaging and Computed Tomography in Emergency Assessment of Patients with Suspected Acute Stroke: a Prospective Comparison. The Lancet 369 (9558), 293-298. doi:10.1016/S0140-6736(07) 60151-2

Chang, L., Yin, C. Y., Wu, H. Y., Tian, B. B., Zhu, Y., Luo, C. X., et al. (2017). (+)-borneol Is Neuroprotective against Permanent Cerebral Ischemia in Rats by Suppressing Production of Proinflammatory Cytokines. J. Biomed. Res. 31 (004), 306-314. doi:10.7555/JBR.31.20160138

Chen, C., Liu, X., and Smith, B. (2003). Utility of Mdr1-Gene Deficient Mice in Assessing the Impact of P-Glycoprotein on Pharmacokinetics and Pharmacodynamics in Drug Discovery and Development. Curr Drug Metab. 4 (4), 272-291. doi:10.2174/1389200033489415

Chen, J. J., Liu, J. C., Chang, L., Zhu, D. Y., and Li, T. Y. (2013). Neuroprotective Effects of Borneol and its Analogues on Glutamate Acid Induced Neuron Injury. J. Nanjing Med. Univ. (Natural Sci. Edition) 33 (5), 630-635. doi:10.7655/NYDXBNS20130513

\section{ACKNOWLEDGMENTS}

YL gratefully acknowledges the support provided by all the members of the scientific research team during the preparation of this manuscript. Special thanks to JW and RM.

Chen, Z, Q., Mou, R. T., Feng, D. X., Wang, Z., and Chen, G. (2017). The Role of Nitric Oxide in Stroke. Med. Gas Res. 7 (3), 194-203. doi:10.4103/2045-9912. 215750

Chen, Z. X., Xu, Q. Q., Shan, C. S., Shi, Y. H., Wang, Y., Chang, R. C. C., et al. (2019). Borneol for Regulating the Permeability of the Blood-Brain Barrier in Experimental Ischemic Stroke: Preclinical Evidence and Possible Mechanism. Oxidative Med. Cell Longevity. 2019, 1-15. doi:10.1155/2019/ 2936737

Cheng, C., Liu, X. W., Du, F. F., Li, M. J., Xu, F., Wang, F. Q., et al. (2013). Sensitive Assay for Measurement of Volatile Borneol, Isoborneol, and the Metabolite Camphor in Rat Pharmacokinetic Study of Borneolum (Bingpian) and Borneolum Syntheticum (Synthetic Bingpian). Acta Pharmacol. Sin. 34, 1337-1348. doi:10.1038/aps.2013.86

Cheng, X. P., Sun, H., and Yu, X. H. (2006). Effect of Borneolum Syntheticum on GABAA Receptor-Mediated Currents in Rat Hippocampal Neurons. Pharmacol. Ctinics Chin. Matetia Mediea 22 (5), 14-16. doi:10.3969/j.issn. 1001-859X.2006.05.008

Chinese Pharmacopoeia Committee (2020). The Pharmacopoeia of People's Republic of China. Beijing, China: China Medical Science Press

Costa, C., Leone, G., Saulle, E., Pisani, F., Bernardi, G., and Calabresi, P. (2004). Coactivation of GABA A and GABA B Receptor Results in Neuroprotection during In Vitro Ischemia. Stroke. 35 (2), 596-600. doi:10.1161/01.STR. 0000113691.32026 .06

De Val, S., and Black, B. L. (2009). Transcriptional Control of Endothelial Cell Development. Developmental Cell 16, 180-195. doi:10.1016/j.devcel.2009. 01.014

Denora, N., Trapani, A., Laquintana, V., Lopedota, A., and Trapani, G. (2009). Recent Advances in Medicinal Chemistry and Pharmaceutical TechnologyStrategies for Drug Delivery to the Brain. Curr Top Med Chem. 9 (2), 182-196. doi: $10.2174 / 156802609787521571$

Dong, T., Chen, N., Ma, X., Wang, J., Wen, J., Xie, Q., et al. (2018). The Protective Roles of L- Borneolum, D- Borneolum and Synthetic Borneol in Cerebral Ischaemia via Modulation of the Neurovascular Unit. Biomed. Pharmacother. 102, 874-883. doi:10.1016/j.biopha.2018.03.087

Duan, S, W., Wang, X., Wang, B., Li, Y. W., and Wang, Y. J. (2012). Effect of Musk, Borneol, Diosgenin and Geniposide on Inflammatory Injury in Cerebral Ischemia and Reperfusion Rats. Pharmacol. Clin. Chin. Materia Med. 28 (03), 43-46. doi:10.13412/j.cnki.zyyl.2012.03.038

Fan, X., Chai, L., Zhang, H., Wang, Y., Zhang, B., and Gao, X. (2015). Borneol Depresses P-Glycoprotein Function by a NF- $\kappa B$ Signaling Mediated Mechanism in a Blood Brain Barrier In Vitro Model. Int J Mol Sci. 16 (11), 27576-27588. doi:10.3390/ijms161126051

Fan, J. J., Li, Y., Fu, X. Y., Li, L. J., Hao, X. T., and Li, S. S. (2017). Nonhuman Primate Models of Focal Cerebral Ischemia. Neural Regen. Res. 12 (2), 321-328. doi:10.4103/1673-5374.200815

Gao, C., Li, X., Li, Y., Wang, L., and Xue, M. (2010). Pharmacokinetic Interaction between Puerarin and Edaravone, and Effect of Borneol on the Brain Distribution Kinetics of Puerarin in Rats. J. Pharm. Pharm. 62, 360-367. doi:10.1211/jpp.62.03.0011

Giannopoulos, S., Kosmidou, M., Hatzitolios, A. I., Savopoulos, C. G., Ziakas, A., and Karamouzis, M. (2008). Measurements of Endothelin-1, C-Reactive Protein and Fibrinogen Plasma Levels in Patients with Acute Ischemic Stroke. Neurol. Res. 30 (7), 727-730. doi:10.1179/174313208X297904

Ginsberg, M. D., and Busto, R. (1998). Combating Hyperthermia in Acute Stroke. Stroke. 29 (2), 529-534. doi:10.1161/01.STR.29.2.529

González-Ibarra, F. P., Varon, J., and López-Meza, E. G. (2011). Therapeutic Hypothermia: Critical Review of the Molecular Mechanisms of Action. Front. Neur. 2, 4. doi:10.3389/fneur.2011.00004

Granger, R. E., Campbell, E. L., and Johnston, G. A. R. (2005). (+)- and (-)-borneol: Efficacious Positive Modulators of GABA Action at Human Recombinant 
a1 $32 \gamma 2 \mathrm{~L}$ GABAA Receptors. Biochem. Pharmacol. 69 (7), 1101-1111. doi:10.1016/j.bcp.2005.01.002

Guo, Y. P. (2000). Pathophysiological Progress of Ischemic Brain Injury and Suggestions for Prevention and Treatment of Cerebral Infarction. Chin. J. Geriatr. Heart Brain Vessel Dis. 002 (006), 368-371. doi:10.3969/j.issn. 1009-0126.2000.06.002

Guo, Y., Yan, S., Xu, L., Zhu, G., Yu, X., and Tong, X. (2014). Use of Angong Niuhuang in Treating Central Nervous System Diseases and Related Research. Evidence-Based Complement. Altern. Med. 2014, 1-9. doi:10.1155/2014/346918

He, X. J. (2005). Study on the Pharmacodynamics of Borneol against Cerebral Ischemia and its Related Mechanism. Shenyang: Shenyang Pharmaceutical University

He, X. J., Zhao, L. M., and Liu, Y. L. (2006). The Protective Effect of Borneol on Experimental Cerebral Ischemia. J. Guangdong Coll. Pharm. 22 (2), 171-173. doi:10.3969/j.issn.1006-8783.2006.02.025

He, X. J., Qin, X. H., and Liu, Y. L. (2007). Effect of Borneol Injection on Cerebral Ischemia. Shanxi Med. J. 036 (09), 794-795. doi:10.3969/j.issn.0253-9926.2007. 17.009

Hsu, Y.-C., Chang, Y.-C., Lin, Y.-C., Sze, C.-I., Huang, C.-C., and Ho, C.-J. (2014). Cerebral Microvascular Damage Occurs Early after Hypoxia-Ischemia via nNOS Activation in the Neonatal Brain. J. Cereb. Blood Flow Metab. 34 (4), 668-676. doi:10.1038/jcbfm.2013.244

Hu, L. M. (2004). Influence of Borneol to Drug Permeability of BBB and Neuroprotective Mechanism of Borneol Combined with Salvianolic Acid B and Saponins of Panax Notoginseng. Tianjin: Tianjin University of Traditional Chinese Medicine

Hu, H., Lu, H., He, Z., Han, X., and Tu, R. (2012). Gene Interference Regulates Aquaporin-4 Expression in Swollen Tissue of Rats with Cerebral Ischemic Edema: Correlation with Variation in Apparent Diffusion Coefficient. Neural Regen. Res. 7 (21), 1659-1666. doi:10.3969/j.issn.1673-5374.2012. 21.009

Hu, L. M., Zhang, Y. J., Wang, W., Wu, Y., Fan, X., Gao, X. M., et al. (2005). Influence of Borneol or Combined with Salvianolic Acid B and Saponins of Panax Notoginseng on the Expression of Vascular Endothelial Growth Factor mRNA in Brain Tissue of Rats with Cerebral Ischemia/reperfusion. Chin. J. Integrated Traditional West. Med. Intensive Crit. Care 12 (05), 263-266. doi:10.3321/j.issn:1008-9691.2005.05.002

Huang, L., Li, Q., Wen, R., Yu, Z., Li, N., Ma, L., et al. (2017). Rho-kinase Inhibitor Prevents Acute Injury against Transient Focal Cerebral Ischemia by Enhancing the Expression and Function of Gaba Receptors in Rats. Eur. J. Pharmacol. 797, 134-142. doi:10.1016/j.ejphar.2017.01.021

Huang, J. Y., Wang, Z. X., Wang, S. X., Guo, H., Chai, L. J., and Hu, L. M. (2020). Protection of Active Pharmaceutical Ingredients in the Xingnaojing Injection against Oxygen Glucose Deprivation/re-Oxygenation-Induced PC12 Cells Injury. Chin. J. Clin. Pharmacol.

Huang, L. (2018). The Same Effect of Beta-Asarone, Borneol, Muscone and Styrax Naphtha about in Inducing Resuscitation and Protecting the Brain. Guangzhou: Guangzhou University of Chinese Medicine

Huang, N., and Zhao, J. (2017). Pathophysiology. second Edition. Bei Jing: Bei Jing Science Press

Huang, P., Jiang, X. F., Zou, J. L., Yuan, Y. M., Yao, M. C., and Lu, Y. S. (2009). A Novel GC-MS Bioanalytical Method for Natural Borneol and its Application in Investigating Natural Borneol Distribution in Mice Mode. Tradit Chin. Med. Mater. Med. 11, 821-827. doi:10.3969/j.issn. 1674-3849.2009.06.012

Hur, J., Pak, S. C., Koo, B.-S., and Jeon, S. (2013). Borneol Alleviates Oxidative Stress via Upregulation of Nrf2 and Bcl-2 in Sh-Sy5y Cells. Pharm. Biol. 51 (1), 30-35. doi:10.3109/13880209.2012.700718

Islam, B. M. N., Uddin, C. J., and Jaripa, B. (2010). Chemical Components in Volatile Oil from Blumea Balsamifera (1.) Dc. Bangladesh J. Bot. 38 (1), 107-109. doi:10.3329/bjb.v38i1.5132

Ito, Y., Ohkubo, T., Asano, Y., Hattori, K., Shimazu, T., Yamazato, M., et al. (2010). Nitric Oxide Production during Cerebral Ischemia and Reperfusion in eNOSand nNOS-Knockout Mice. Curr Neurovasc Res. 7 (1), 23-31. doi:10.2174/ 156720210790820190

Jia, Y. L. (2014). Effects of Borneol on Rat's Blood Brain Barrier and Zonula Occludens-1 after Global Cerebra Ischemia-Reperfusion Injury. Xinxiang: Xinxiang Medical University
Jiang, X. F., Zou, J. L., Yuan, Y. M., Francis, C. P. Law., Qiao, Y. J., and Yao, M. C. (2008). Preliminary Study: Biotransformation of Borneol to Camphor in Mice, Rats, and Rabbits. World Sci. Technology. 10 (3), 27-36. doi:10.1016/S18763553(09)60013-2

Kawabori, M., and Yenari, M. (2015). Inflammatory Responses in Brain Ischemia. Curr Med Chem. 22 (10), 1258-1277. doi:10.2174/0929867322666150209154036

Ke, Y. Q., Xu, R, X., and Chen, C. C. (2000). Effect of 5-Hydroxytryptamine Level Changes on Permeability of Blood-Brain Barrier Following Brain Injury. Chin. J. Clin. Neurosurg. 5 (z1), 40-42. doi:10.3969/j.issn.1009-153X.2000.z1.015

Kong, Q., Wu, Z., Chu, X., Liang, R., Xia, M., and Li, L. (2013). Study on the Anticerebral Ischemia Effect of Borneol and its Mechanism. Afr. J. Trad. Compl. Alt. Med. 11 (1), 161-4. doi:10.4314/ajtcam.v1li1.25

Lai, T. W., Zhang, S., and Wang, Y. T. (2014). Excitotoxicity and Stroke: Identifying Novel Targets for Neuroprotection. Prog. Neurobiol. 115, 157-188. doi:10.1016/ j.pneurobio.2013.11.006

Li, J. M., Hu, S. X., and Li, H. Q. (2013). The Commodity Types and Historical Origins of Traditional Chinese Medicine Borneol. Modern Chinese Medicine. doi:10.3969/j.issn.1673-4890.2013.06.023

Li, Y, J. (2016). The Molecular Mechanisms of Left-Handed Camphor Prevents Autophagy by Targeting miR-140 in PC12 Cells. Guangzhou: Guangzhou University of Chinese Medicine

Li, Y. (2018). Mechanism Study of Natural Borneol in Treatment of Ischemic Stroke. Tai'an: Taishan Medical University. doi:10.27353/d.cnki.gtsyc.2018.000151

Li, Y. H., Sun, X. P., Zhang, Y. Q., and Wang, N. S. (2008). The Antithrombotic Effect of Borneol Related to its Anticoagulant Property. Am. J. Chin. Med. 36 (04), 719-727. doi:10.1142/S0192415X08006181

Li, X., Wan, X., Gao, J. J., Zhao, C. S., Liu, X. F., Yang, L. J., et al. (2017). Effects of Huazhuo Jiedu Huoxue Tongluo Prescription on Expression of Tight Junction ZO-1 and Occludin in Rats with Cerebral Ischemia Reperfusion Injury. Global Traditional Chinese Medicine. doi:CNKI:SUN:HQZY.0.2017-07-005

Liang, Z. W., Dong, Q. F., Wang, M. M., Gao, K., Li, J. K., and Yang, L. (2016). Protective Effects of Borneol "Meridian Guiding" Salvia Miltiorrhiza in Treatment of Cerebral Ischemia/Reperfusion. Prog. Mod. Biomed. 16 (13), 2430-2433. doi:10.13241/j.cnki.pmb.2016.13.007

Liao, S., Han, L., Zheng, X., Wang, X., Zhang, P., Wu, J., et al. (2019). Tanshinol Borneol Ester, a Novel Synthetic Small Molecule Angiogenesis Stimulator Inspired by Botanical Formulations for Angina Pectoris. Br. J. Pharmacol. doi:10.1111/bph.14714

Liu, H. T., and Mu, D. Z. (2014). [Inducible Nitric Oxide Synthase and Brain Hypoxic-Ischemic Brain Damage]. Zhongguo Dang Dai Er Ke Za Zhi. 16 (9), 962-967. Chinese. doi:10.7499/j.issn.1008-8830.2014.09.022

Liu, Y. Q. (2017). L-camphor Target MicroRNA-125-3p on the Mechanism of Cerebral Ischemia Reperfusion Injury. Guangzhou University of Traditional Chinese Medicine

Liu, Y. M., Zhang, C. A., Xu, Q. Y., and Peng, S. Q. (2002a). Effect of Moschus and Borneol on the Content of Aminoacid Neurotransmitter in Brain Tissue of Rats with Cerebral Ischemia and Reperfusion Injury. Traditional Chin. Drug Res. Clin. Pharmacol. (04), 231-233. doi:10.3321/j.issn:1003-9783.2002.04.013

Liu, Y. M., Zhang, Z. A., Xu, Q. Y., Zeng, Z. L., and Peng, S. Q. (2002b). The Influence on the Brain Edema, SOD and MDA of Ischemia Reperfusion Rat by Using Inducing Resuscitation Method. Chin. J. Inf. Traditional Chin. Med. 009 (7), 22-24. doi:10.3969/j.issn.1005-5304.2002.07.010

Liu, Y. M., Zhao, G. F., Xia, X. H., Xu, Q. Y., and Shen, Q. (2009). Influence of Muskiness Compounded with Borneol on Blood Brain Barrier after Ischemic Reperfusion Injury. Beijing J. Traditional Chin. Med. 28 (06), 459-462. doi: CNKI:SUN:BJZO.0.2009-06-025

Liu, R., Zhang, L., Lan, X., Li, L., Zhang, T.-T., Sun, J.-H., et al. (2011). Protection by Borneol on Cortical Neurons against Oxygen-Glucose Deprivation/reperfusion: Involvement of Anti-oxidation and Anti-inflammation through Nuclear Transcription Factor карраB Signaling Pathway. Neuroscience. 176, 408-419. doi:10.1016/j.neuroscience.2010.11.029

Liu, Y. M., Xia, X. H., Zhao, G. F., Peng, S. Q., Xu, Q. Y., and Shen, Q. (2007). Effect of Moschus Combined with Borneol on Brain Water Content and Blood-Brain Barrier Permeability in Rat Model of Cerebral Focal Ischemia with Reperfusion. J. Guangzhou Univ. Traditional Chin. Med. 24 (6), 498-501. doi:10.3969/j.issn. 1007-3213.2007.06.016

Liu, L., Wei, H., and Xu, Li. (2018). Effects of Different Doses of Musk and Borneol on the Neurological Deficit Score,activities of COX-2 and 5-LOX in Brain and 
CysLT2 Expression in the hippocampus of Cerebral IR Model. J. Clin. Exp. Med. 17 (12), 1241-1244. doi:10.3969/j.issn.1671-4695.2018.12.003

Lu, Y., Du, S.-y., Chen, X.-l., Wu, Q., Song, X., Xu, B., et al. (2011). Enhancing Effect of Natural Borneol on the Absorption of Geniposide in Rat via Intranasal Administration. J. Zhejiang Univ. Sci. B. 12 (2), 143-148. doi:10.1631/jzus. B1000121

Luo, S. L., Wang, J., Wen, J., Cao, C., and Xie, Q. (2016). Monitoring the Impact of Three Borneol Temperature Fever Rats Dynamic Concept Based on TCM. Lishizhen Med. Materia Med. Res. 27 (3), 521-523. doi:10.3969/j.issn.10080805.2016.03.004

Lv, X. X., Sun, M. J., and Sun, F. Z. (2012). [Research of Bornrol Promote Drugs through Blood-Brain Barrier]. Zhongguo Zhong Yao Za Zhi. 37 (7), 878-881. doi:10.4268/cjcmm20120702

Ma, R., Xie, Q., Li, H., Guo, X., Wang, J., Li, Y., et al. (2021). 1-Borneol Exerted the Neuroprotective Effect by Promoting Angiogenesis Coupled with Neurogenesis via Ang1-VEGF-BDNF Pathway. Front. Pharmacol. 12. doi:10.3389/fphar. 2021.641894

Ma, R., Xie, Q., Li, Y., Chen, Z., Ren, M., Chen, H., et al. (2020). Animal Models of Cerebral Ischemia: a Review. Biomed. Pharmacother. 131, 110686. doi:10.1016/ j.biopha.2020.110686

Mcbride, D. W., and Zhang, J. H. (2017). Precision Stroke Animal Models: the Permanent MCAO Model Should Be the Primary Model, Not Transient MCAO. Transl. Stroke Res. 8 (5), 397-404. doi:10.1007/s12975-0170554-2

Mele, M., Ribeiro, L., Inácio, A. R., Wieloch, T., and Duarte, C. B. (2014). GABAA Receptor Dephosphorylation Followed by Internalization Is Coupled to Neuronal Death in In Vitro Ischemia. Neurobiol. Dis. 65, 220-232. doi:10. 1016/j.nbd.2014.01.019

Mendes, R. T., Nguyen, D., Stephens, D., Pamuk, F., Fernandes, D., Hasturk, H., et al. (2018). Hypoxia-induced Endothelial Cell Responses - Possible Roles during Periodontal Disease. Clin. Exp. Dent Res. 4, 241-248. doi:10.1002/ cre2.135

Michael, S., Ignaz, G., Christoph, K., and Peter, K. (2016). Immunohistochemical Analysis of Cerebral Thrombi Retrieved by Mechanical Thrombectomy from Patients with Acute Ischemic Stroke. Int. J. Mol. Sci. 17 (3), 298. doi:10.3390/ ijms 17030298

Ni, C. X. (2011). The Effects of Aromatic Resuscitation Drugs on Anti-brain Ischemia and the Function of Blood-Brain Barrier. Chengdu: Chengdu University of traditional Chinese Medicine

Pan, X. U., Ying, L. I., Shou-Ying, D. U., Yang, L. U., Bai, J., and Guo, Q. L. (2014). Comparative Pharmacokinetics of Borneol in Cerebral Ischemia-Reperfusion and Sham-Operated Rats. J. Zhejiang Universityence B. 15 (1), 84-91. doi:10.1631/jzus.B1300141

Rama, R., and García., J. C. (2016). Excitotoxicity and Oxidative Stress in Acute Stroke. Excitotoxicity and Oxidative Stress in Acute Stroke. Acute Ischemic Stroke. InTech

Ren, Z. X. (2018). miR-140-HnrnpAl Inhibits Stress Granule Formation and L-Camphor Intervention. Guangzhou: Guangzhou University of Chinese Medicine

Santos, S. E., Ribeiro, F. P. R. A., Menezes, P. M. N., Duarte Filho, L. A. M., Quintans, J. S. S., Quintans Junior, L. J., et al. (2018). New Insights on Relaxant Effects of (-)-borneol Monoterpenein Rat Aortic Rings. Fundam. Clin. Pharmacol. 33. doi:10.1111/fcp.12417

Shang, K., Sui, D. J., Liu, Q. M., Wang, D. Y., Zhang, X., and Qiu, Z. D. (2015). Effects of Pair Herbs "Yanhusuo-bingpian" on Cerebral Blood Flow Dynamics in Anesthetized Dogs. Chin. J. Vet. Sci. (06), 153-156. doi:CNKI:SUN: ZSYX.0.2015-06-030

Shao, X. R., Cai, K. R., Jia, R., Zhao, H., Li, L., Zhou, X., et al. (2018). Effects of Borneol on Inflammatory Reaction and Permeability of Blood-Brain Barrier in Rats with Cerebral Ischemic Injury. Chin. J. Clin. Pharmacol. 34 (13). 1558-1560. doi:10.13699/j.cnki.1001-6821.2018.13.020

Shi, Y. M. (2014). Research Progress of Calcium Channels in the Neuron after Cerebral Ischemia/Reperfusion Injury. Med. Recapitulate 20 (14), 2507-2509. doi:10.3969/j.issn.1006-2084.2014.14.005

Shimotake, J., Derugin, N., Wendland, M., Vexler, Z. S., and Ferriero, D. M. (2010). Vascular Endothelial Growth Factor Receptor-2 Inhibition Promotes Cell Death and Limits Endothelial Cell Proliferation in a Neonatal Rodent
Model of Stroke. Stroke. 41, 343-349. doi:10.1161/STROKEAHA.109. 564229

Sims, N. R., and Yew, W. P. (2017). Reactive Astrogliosis in Stroke: Contributions of Astrocytes to Recovery of Neurological Function. Neurochem. Int. 107, 88-103. doi:10.1016/j.neuint.2016.12.016

Singh, D. P., and Chopra, K. (2014). Flavocoxid, Dual Inhibitor of Cyclooxygenase2 and 5-lipoxygenase, Exhibits Neuroprotection in Rat Model of Ischaemic Stroke. Pharmacol. Biochem. Behav. 120, 33-42. doi:10.1016/j.pbb.2014.02.006

Singh, V., Mishra, V. N., Chaurasia, R. N., Joshi, D., and Pandey, V. (2019). Modes of Calcium Regulation in Ischemic Neuron. Ind. J. Clin. Biochem. 34 (2), 246-253. doi:10.1007/s12291-019-00838-9

Steliga, A., Kowiański, P., Czuba, E., Waśkow, M., Moryś, J., and Lietzau, G. (2020). Neurovascular Unit as a Source of Ischemic Stroke Biomarkers-Limitations of Experimental Studies and Perspectives for Clinical Application. Transl. Stroke Res. 11, 553-579. doi:10.1007/s12975-019-00744-5

Sun, B. Z., Chen, L., Wu, Q., Wang, H. L., Wei, X. B., Xiang, Y. X., et al. (2014). Suppression of Inflammatory Response by Flurbiprofen Following Focal Cerebral Ischemia Involves the NF-Kb Signaling Pathway. Int. J. Clin. Exp. Med. 7 (9), 3087-3095.

Sun, S. P., Du, Y. Y., Suo, X. G., Wang, Y., Zhang, J. H., Wu, C. G., et al. (2019). Effect of Borneol on Lipopolysaccharide-Induced RAW264.7 Macrophage Inflammation Model. J. Tonghua Normal Univ. 40 (4)

Tang, Y. X. (2010). The Experimental Study on the Anti-Acute Brain Isehemia Effect of Four Aromatic Resuscitation Drugs and the Initial Exploring The Drug Property. Chengdu: Chengdu University of traditional Chinese Medicine

Tian, H. (2013). Comparative Research on Mechanism of Neuroprotective Effect and Influence of Blood-Brain Barrier between L-Borneolum and Bomeolum Svntheticum. Chengdu: Chengdu University of Traditional Chinese Medicine

Tu, X.-k., Yang, W.-z., Shi, S.-s., Wang, C.-h., Zhang, G.-l., Ni, T.-r., et al. (2010). Spatio-Temporal Distribution of Inflammatory Reaction and Expression of TLR2/4 Signaling Pathway in Rat Brain Following Permanent Focal Cerebral Ischemia. Neurochem. Res. 35 (8), 1147-1155. doi:10.1007/s11064-010-0167-6

Ueno, M. (2009). Mechanisms of the Penetration of Blood-Borne Substances into the Brain. Curr Neuropharmacol. 7 (2), 142-149. doi:10.2174/ 157015909788848901

Ueno, M., Nakagawa, T., Wu, B., Onodera, M., Huang, C.-1., Kusaka, T., et al. (2010). Transporters in the Brain Endothelial Barrier. Curr Med Chem. 17, 1125-1138. doi:10.2174/092986710790827816

Vannucci, R. C., Brucklacher, R. M., and Vannucci, S. J. (2001). Intracellular Calcium Accumulation during the Evolution of Hypoxic-Ischemic Brain Damage in the Immature Rat. Developmental Brain Res. 126 (1), 117-20. doi:10.1016/s0165-3806(00)00135-8

Wang, Y. (2011). The Effect of Musk,Borneol and Compatibilitv of Them Onn the Animal Models of Cerebral Ischemia. Chengdu: Chengdu University of Traditional Chinese Medicine

Wang, L. Y., Liu, J., Li, Y., Li, B., Zhang, Y. Y., Jing, Z. W., et al. (2015a). Timedependent Variation of Pathways and Networks in a 24-hour Window after Cerebral Ischemia-Reperfusion Injury. BMC Syst. Biol. 9 (1), 11. doi:10.1186/ s12918-015-0152-4

Wang, N., Ma, H. P., Qi, X. Z., Meng, P., and Jia, Z. P. (2015b). Progression of Nrf2ARE Signaling Pathway in Protection of Oxidative Stress Injury of the Body. Med. Pharm. J. Chin. People's Liberation Army 27 (12), 21-27. doi:10.3969/j. issn.2095-140X.2015.12.005

Wang, Z.-P., Tian, Y., and Lin, J. (2017b). Role of Wild-type P53-Induced Phosphatase 1 in Cancer. Oncol. Lett. 14, 3893-3898. doi:10.3892/ol.2017.6685

Wang, J., Qu, X. L., Fan, C. D., and Gao, H. L. (2018). Study on Action Mechanism of Borneol Combined with Safflower on Cerebral Ischemia Injury in Rats. Chin. J. Hosp. Pharm. 038 (023), 2410-2415. doi:10.13286/j.cnki.chinhosppharmacyj. 2018.23.06

Wang, L., Liang, Q., Lin, A., Wu, Y., Min, H., Song, S., et al. (2019). Borneol Alleviates Brain Injury in Sepsis Mice by Blocking Neuronal Effect of Endotoxin. Life Sci. 232, 116647. doi:10.1016/j.lfs.2019.116647

Wang, J., and Wang, S. Y. (2018). Chinese Herbal Medicine. Beijing: China Medical Science Press

Wen, J. (2017). Research on Protection Mechanism of Aromatic Resuscitation Drug against Neurovascular Unit in Permanent Cerebral Ischemia Model Rats. Chengdu: Chengdu University of traditional Chinese Medicine 
Wen, J., Wang, J., Luo, S, L., Peng, W., and Ren, Y. M. (2016). Advances in Studies on Regulating Effects of Wnt/ $\beta$-Catenin Signaling Pathway on Neurovascular Unit after Cerebral Ischemia and Related Medicine. Chin. Pharmacol. Bull. 32 (03), 310-314. doi:10.3969/j.issn.1001-1978.2016.03.003

Wermuth, C. G. (2015). Chapter 18 - Optical Isomerism in Drugs. The Practice of Medicinal Chemistry (Fourth Edition). Salt Lake: Academic Press, 429-447.

Wöckel, L., Koch, S., Stadler, C., Meyer-Keitel, A.-E., and Schmidt, M. (20082008). Serotonin-induced Platelet Intracellular Ca2+ Response in Patients with Anorexia Nervosa. Pharmacopsychiatry 41 (1), 10-16. doi:10.1055/s-2007992145

Wrotek, S. E., Kozak, W. E., Hess, D. C., and Fagan, S. C. (2011). Treatment of Fever after Stroke: Conflicting Evidence. Pharmacotherapy 31 (11), 1085-1091. doi:10.1592/phco.31.11.1085

Wu, C., Liao, Q., Yao, M., Xu, X., Zhou, Y., Hou, X., et al. (2014a). Effect of Natural Borneol on the Pharmacokinetics and Distribution of Nimodipine in Mice. Eur. J. Drug Metab. Pharmacokinet. 39, 17-24. doi:10.1007/s13318013-0135-Z

Wu, H.-Y., Tang, Y., Gao, L.-Y., Sun, W.-X., Hua, Y., Yang, S.-B., et al. (2014b). The Synergetic Effect of Edaravone and Borneol in the Rat Model of Ischemic Stroke. Eur. J. Pharmacol. 740, 522-531. doi:10.1016/j.ejphar.2014.06.035

Wu, J. J., Wang, H. J., Yang, S., Liu, Y., and Xu, X. Y. (2016). [Borneol Promotes Catalpol and Puerarin Crossing through Blood-Brain Barrier in Focal Cerebral Ischemic Rats]. Zhongguo Zhong Yao Za Zhi. 41 (21), 3988-3995. doi:10.4268/ cjcmm20162117

Xiao, Y. Y., Ping, Q. N., and Chen, Z. P. (2007). The Enhancing Effect of Synthetical Borneol on the Absorption of Tetramethylpyrazine Phosphate in Mouse. Int. J. Pharm. 337 (1-2), 74-79. doi:10.1016/j.ijpharm.2006.12.034

Xing, H. Y., Sun, S. G., Mei, Y. W., Shi, J., and Dirk, M. H. (2007). Inhibition of P-Glycoprotein Potentiates Neuroprotection of FK506 on Ischemic Brain Injury. CHINESE JOURNAL NEUROLOGY. 40 (2), 112-115. doi:10.3760/j. issn:1006-7876.2007.02.011

Xu, J., Li, C., Yin, X.-H., and Zhang, G.-Y. (2008). Additive Neuroprotection of Gaba a and Gaba B Receptor Agonists in Cerebral Ischemic Injury via Pi-3k/akt Pathway Inhibiting the Ask1-Jnk Cascade. Neuropharmacology. 54 (7), 1029-1040. doi:10.1016/j.neuropharm.2008.01.014

Xu, L., and Zhang, T. J. (2016). Effects of Borneolum Syntheticum Combined with Breviscapine on Blood-Brain Barrier Permeability Induced by Hypoxia/ Reoxygenation Injury. Chin. J. Inf. Traditional Chin. Med. 23 (02), 76-78. doi:10.3969/j.issn.1005-5304.2016.02.021

$\mathrm{Xu}, \mathrm{L}$., and Zhang, T. J. (2015). Effects of Borneol Combined with Breviscapine on Blood-Brain Barrier Permeability after Ischemia Reperfusion Injury in Rats. Pharmacol. Clin. Chin. Materia Med. 31 (1), 74-76. doi:CNKI:SUN: ZYYL.0.2015-01-025

Xu, Y. B., Mao, X. N., Xu, G. C., Tao, L. D., Zhang, J., and Zhang, P. J. (2016). The Role of Apoptosis Proteins Bcl-2, Bax in Hepatic Ischemia-Reperfusion Injury Model. Chin. J. Clinicians (Electronic Edition). 010 (13), 1968-1971. doi:10.3877/cma.j.issn.1674-0785.2016.13.026

Yan, L., Hu, J. P., Shao, X. R., Sun, X. D., and C, K. R. (2018). Effects of Icariin Combined with Borneol on Antioxidant Activity Inflammatory Reaction and Permeability of Blood-Brain Barrier in Rats Following Cerebral Ischemic Injury. Shaanxi J. Tradit. Chin. Med. 39 (12), 5-8. doi:CNKI:SUN: SXZY.0.2018-12-001

Yang, L., Li, W. R., Kui, S. Q., and Wang, N. S. (2010). Inhibitory Effects and Mechanism of Bornel on Arterial Thrombosis Induced by $\mathrm{FeCl} 3$ in Rat. Chin. J. Exp. Traditional Med. Formulae. 16 (006), 164-166. doi:10.3969/j.issn.10059903.2010.06.052

Yang, X. Q., Ding, H., Liu, X. D., Tang, S., Yang, R. Y., Deng, C. Q., et al. (2019). Study on Borneol Combined with Astragaloside IV and Panax Notoginseng Saponins Promoting Nerve Repair after Cerebral Ischemia-Reperfusion. China J. Traditional Chin. Med. Pharm.

Yang, Z., Guo, P., Han, R., Wu, S., and Gao, J.-M. (2018). Gram-scale Separation of Borneol and Camphor from Cinnamomum Camphora (L.) Presl by Continuous Counter-current Chromatography. Sep. Sci. Plus 1, 144-153. doi:10.1002/sscp. 201700041

Yao, H. W., Wang, J., Liu, Y., and Wang, D. D. (2011). Effect of Moschus and Borneo Camphor in Different Proportion on Bloodbrain Barrier Permeability of Acute Cerebral Ischemic Model Mice. J. Chengdu Univ. Traditional Chin. Med.
Yoo, K.-Y., Hwang, I. K., Lee, C. H., Choi, J. H., Kwon, S.-H., Kang, I.-J., et al. (2010). Difference of Fibroblast Growth Factor Receptor 1 Expression Among CA1-3 Regions of the Gerbil hippocampus after Transient Cerebral Ischemia. J. Neurol. Sci. 296, 13-21. doi:10.1016/j.jns.2010.06.018

Yu, B., Lu, G. H., Sun, Y., Lin, X., and Fang, T. H. (2011). Effect of Electroacupuncture Combined with Intragastric Administration of Borneol on the Permeability of Blood-Brain Barrier in the Mouse. Acupuncture Res. 36 (05), 335-340.

Yu, B., Ruan, M., Cui, X. B., Guo, J. M., Xu, L., and Dong, X. P. (2013). Effects of Borneol on the Pharmacokinetics of Geniposide in Cortex, hippocampus, Hypothalamus and Striatum of Conscious Rat by Simultaneous Brain Microdialysis Coupled with UPLC-MS. J. Pharm. Biomed. Anal. 77, 128-132. doi:10.1016/j.jpba.2013.01.017

Yu, B., Ruan, M., Zhang, Z. N., Cheng, H. B., and Shen, X. C. (2016). Synergic Effect of Borneol and Ligustrazine on the Neuroprotection in Global Cerebral Ischemia/reperfusion Injury: a Region-Specificity Study. Evid Based. Complement. Altern. Med. 2016, 1-8. doi:10.1155/2016/4072809

Yu, B., Liang, T., Huang, S. W., and Li, Y. (2017a). The Anti-ischemia Effect of Different Dose of Borneol Combining with Chuanxiong Rhizoma on the Global Cerebral Ischemia Rats in Different Brain Regions. Chin. J. Gerontol. 37 (10), 2352-2355. doi:10.3969/j.issn.1005-9202.2017.10.005

Yu, B., Ruan, M., Liang, T., Huang, S. W., Yu, Y., Cheng, H. B., et al. (2017b). The Synergic Effect of Tetramethylpyrazine Phosphate and Borneol for Protecting against Ischemia Injury in Cortex and hippocampus Regions by Modulating Apoptosis and Autophagy. J. Mol. Neurosci. 63 (1), 70-83. doi:10.1007/s12031017-0958-1

Yu, B., Shen, X. C., Liang, T., Huang, S. W., Yang, L., Cheng, H. B., et al. (2017c). Research on the Synergy of Different Doses of Borneol and Chuanxiong Rhizoma on the Anti-ischemnia Effect in Different Brain Regions. Chin. J. Hosp. Pharm. 37 (18), 1792-1796. doi:10.13286/j.cnki.chinhosppharmacyj. 2017.18.05

Yu, B., Ruan, M., Xu, L., Liu, S. J., Xu, H. Q., and Sheng, X. C. (2019a). Synergic Effect of Chuanxiong Rhizoma and Borneol on Protecting hippocampus and Hypothalamusof Rats from Global Cerebral Ischemia Reperfusion Injury. Chin. Pharmacol. Bull. 35 (9), 1302-1307. doi:10.3969/j.issn.1001-1978.2019.09.022

Yu, B., Zhong, F. M., Yao, Y., Deng, S. Q., Xu, H. Q., Lu, J. F., et al. (2019b). Synergistic Protection of Tetramethylpyrazine Phosphate and Borneol on Brain Microvascular Endothelium Cells Injured by Hypoxia. Am. J. Transl Res. 11 (4), 2168-2180.

Yu, B., Ruan, M., Liang, T., and Yu, Y. (2020). Synergy between Borneol and Extract of Ligusticum Chuanxiong Hort against Cortex and Striatum Ischemia. Int. J. Pharmacol. 16, 104-119. doi:10.3923/ijp.2020.104.119

Yuan, Z., Zhang, J. P., Liu, Y. F., and Zhang, B. L. (2006). Study on the Relationship between P-Glycoprotein and Borneol Promoting the Physiological Opening of Blood-Brain Barrier. Tianjin J. Traditional Chin. Med. 023 (03), 261-262. doi:10.3969/j.issn.1672-1519.2006.03.041

Zhang, C. A., Liu, Y. M., Xu, Q. Y., and Peng, S. Q. (2002). Effect of Aromatic Herbs for Resuscitation on NO Content and Expression of NOS in Brain Tissue of Rats with Cerebral Schemia and Reperfusion Injury. J. Guangzhou Univ. Traditional Chin. Med. 19 (02), 115-118. doi:10.3969/j.issn.1007-3213.2002.02.011

Zhang, X. Y. (2013). Cysteinyl Leukotriene CysLT2 Receptor Mediates Neuronal Injury Damage through Activation Microglia. Hangzhou: Zhejiang University Zhang, Q., Wu, D., Wu, J., Ou, Y., Mu, C., Han, B., et al. (2015). Improved BloodBrain Barrier Distribution: Effect of Borneol on the Brain Pharmacokinetics of Kaempferol in Rats by In Vivo Microdialysis Sampling. J. Ethnopharmacology. 162, 270-277. doi:10.1016/j.jep.2015.01.003

Zhang, Q.-L., Fu, B. M., and Zhang, Z.-J. (2017a). Borneol, a Novel Agent that Improves Central Nervous System Drug Delivery by Enhancing Blood-Brain Barrier Permeability. Drug Deliv. 24 (1), 1037-1044. doi:10.1080/10717544. 2017.1346002

Zhang, X.-G., Shan, C., Zhu, J.-Z., Bao, X.-Y., Tong, Q., Wu, X.-F., et al. (2017b). Additive Neuroprotective Effect of Borneol with Mesenchymal Stem Cells on Ischemic Stroke in Mice. Front. Physiol. 8, 1133. doi:10.3389/fphys.2017.01133

Zhang, Y.-M., Qu, X.-Y., Tao, L.-N., Zhai, J.-H., Gao, H., Song, Y.-Q., et al. (2019). XingNaoJing Injection Ameliorates Cerebral Ischaemia/reperfusion Injury via SIRT1-Mediated Inflammatory Response Inhibition. Pharm. Biol. 58 (9), 16-24. doi:10.1080/13880209.2019.1698619 
Zhao, B. S., Xu, Q., Mi, S. Q., and Wang, N. S. (2001). The Relationship between eNOS and the Function of Borneol in Stimutating the BBB`s Opening. J. Brain Nervous Dis. 9 (4), 207-209. doi:10.3969/j.issn.1006-351X.2001.04.006

Zhao, J.-y., Lu, Y., Du, S.-y., Song, X., Bai, J., and Wang, Y. (2012). Comparative Pharmacokinetic Studies of Borneol in Mouse Plasma and Brain by Different Administrations. J. Zhejiang Univ. Sci. B 13, 990-996. doi:10.1631/jzus.B1200142

Zhong, M., Chen, H., Jiang, Y., Tu, P. F., Liu, C. L., Zhang, W. X., et al. (2012). Effect of Echinacoside on Striatal Extracellular Level of Amino Acids Neurotransmitter in Cerebral Ischemia Rats. Chin. Pharmacol. Bull. (3), 361-365. doi:10.3969/j.issn.1001-1978.2012.03.015

Zhou, L., and Zhu, D.-Y. (2009). Neuronal Nitric Oxide Synthase: Structure, Subcellular Localization, Regulation, and Clinical Implications. Nitric Oxide 20, 223-230. doi:10.1016/j.niox.2009.03.001

Zou, L., Zhang, Y., Li, W., Zhang, J., Wang, D., Fu, J., et al. (2017). Comparison of Chemical Profiles, Anti-inflammatory Activity, and
UPLC-Q-TOF/MS-Based Metabolomics in Endotoxic Fever Rats between Synthetic Borneol and Natural Borneol. Molecules. 22 (9), 1446. doi:10.3390/molecules22091446

Conflict of Interest: The authors declare that the research was conducted in the absence of any commercial or financial relationships that could be construed as a potential conflict of interest.

Copyright (C) $2021 \mathrm{Li}$, Ren, Wang, Ma, Chen, Xie, Li, Li and Wang. This is an openaccess article distributed under the terms of the Creative Commons Attribution License (CC BY). The use, distribution or reproduction in other forums is permitted, provided the original author(s) and the copyright owner(s) are credited and that the original publication in this journal is cited, in accordance with accepted academic practice. No use, distribution or reproduction is permitted which does not comply with these terms. 


\section{GLOSSARY}

5-HT 5-hydroxytryptamine

5-LOX 5-lipoxygenase

APC adenomatous polyposis coli

BBB blood-brain barrier

BDNF brain-derived neurotrophic factor

bFGF/FGF2 fibroblast growth factor 2

BMEC brain micro-vascular endothelial cell

Cadm2 bell adhesion molecule 2

CAT Catalase

CGRP calcitonin gene-related peptide

CIR cerebral ischemia reperfusion

CNS central nervous system

COX-2 cyclooxygenase-2

CysLT-2 cysteinyl leukotriene receptor-2

Dsh disheveled

EB evans blue

eNOS endothelial nitric oxide synthase

ET-1 endothelin-1

GABA gamma-aminobutyric acid

GDNF glial cell-derived neurotrophic factor

Glu glutamic acid

GSH-Px glutathione peroxidase

GSK-3 $\beta$ glycogen synthase kinase-3 beta

$\mathbf{H}_{2} \mathbf{O}_{2}$ Hydrogen peroxide

HnrnpA1 heterogeneous Nuclear Ribonucleoprotein A1

ICAM-1 intercellular adhesion molecule-1

IL-10 interleukin-10

IL-1 $\beta$ interleukin- $1 \beta$

IL-6 interleukin-6

iNOS inducible nitric oxide synthase

LC3 microtubule-associated protein light chain 3

LEF1 lymphoid Enhancer-Binding Factor 1
LFA-1 lymphocyte function-associated antigen-1

MDA malondialdehyde

MMP 2 matrix metallopeptidase 2

MMP 9 matrix metallopeptidase 9

MPO Myeloperoxidase

mTOR mammalian Target of rapamycin

NF- $\mathbf{k B}$ nuclear factor- $\mathrm{\kappa B}$

NGF nerve growth factor

NMDA-R N-methyl-d-aspartate glutamate receptor

nNOS neuronal nitric oxide synthase

NO nitric oxide

NOS nitric oxide synthase

$\mathbf{O}^{2-}$ superoxide anion

OGD/R Oxygen-Glucose Deprivation/Reperfusion

$\mathbf{O H}^{-}$hydroxyl radical

p53: tumor protein 53

p62 sequestosome-1

pBCO permanent bilateral carotid occlusion

pCIR permanent cerebral ischemia reperfusion

P-gp P-glycoprotein

ROS reactive oxygen species

SOD superoxide dismutase

tBCO transient bilateral carotid occlusion

TCM traditional Chinese Medicine

TTC 2,3,5-Triphenyltetrazolium chloride

tGCIR transient Global cerebral ischemia reperfusion

TIMP-1 tissue inhibitor of metalloproteinase-1

tMCAO transient middle cerebral artery occlusion

TNF- $\boldsymbol{\alpha}$ necrosis factor- $\alpha$

VEGF vascular endothelial growth factor

VEGFR1/FLT1 fms related receptor tyrosine kinase 1

VEGFR2/KDR Kinase insert domain receptor

Wnt3a wnt family member 3A 